\title{
Potential Vorticity and Balanced and Unbalanced Moisture
}

\author{
Alfredo N. Wetzel \\ Department of Mathematics, University of Wisconsin-Madison, Madison, Wisconsin
}

LESLIE M. SMITH

Department of Mathematics, and Department of Engineering Physics, University of Wisconsin-Madison, Madison, Wisconsin

SAMUEL N. STECHMANN

Department of Mathematics, and Department of Atmospheric and Oceanic Sciences, University of Wisconsin-Madison, Madison, Wisconsin

\section{JONATHAN E. MARTIN}

Department of Atmospheric and Oceanic Sciences, University of Wisconsin-Madison, Madison, Wisconsin

\author{
YEYU ZHANG \\ Department of Mathematics, University of Wisconsin-Madison, Madison, Wisconsin
}

(Manuscript received 6 November 2019, in final form 9 March 2020)

\begin{abstract}
Atmospheric flows are often decomposed into balanced (low frequency) and unbalanced (high frequency) components. For a dry atmosphere, it is known that a single mode, the potential vorticity (PV), is enough to describe the balanced flow and determine its evolution. For a moist atmosphere with phase changes, on the other hand, balanced-unbalanced decompositions involve additional complexity. In this paper, we illustrate that additional balanced modes, beyond PV, arise from the moisture. To support and motivate the discussion, we consider balanced-unbalanced decompositions arising from a simplified Boussinesq numerical simulation and a hemispheric-sized channel simulation using the Weather Research and Forecasting (WRF) Model. One important role of the balanced moist modes is in the inversion principle that is used to recover the moist balanced flow: rather than traditional PV inversion that involves only the PV variable, it is $\mathrm{PV}$-and- $M$ inversion that is needed, involving $M$ variables that describe the moist balanced modes. In examples of PV-and- $M$ inversion, we show that one can decompose all significant atmospheric variables, including total water or water vapor, into balanced (vortical mode) and unbalanced (inertio-gravity wave) components. The moist inversion, thus, extends the traditional dry PV inversion to allow for moisture and phase changes. In addition, we illustrate that the moist balanced modes are essentially conserved quantities of the flow, and they act qualitatively as additional PV-like modes of the system that track balanced moisture.
\end{abstract}

\section{Introduction}

Meteorologically significant midlatitude motions are principally associated with flows that are in near-geostrophic balance (rapid rotation and strong stratification). This balanced flow acts somewhat independently of the

Corresponding author: Alfredo N. Wetzel, alfredo.wetzel@ wisc.edu transient high-frequency inertio-gravity and acoustic waves. Balanced motion is, therefore, primarily low frequency and synoptic in scale.

Accordingly, to discern significant and long-lasting motions, it is often beneficial to decompose atmospheric flow into its balanced and unbalanced components. In the dry atmosphere, such a decomposition may be carried out through the identification of the low-frequency vortical mode of the flow to construct a single potential 
vorticity (PV) variable determining the evolution of the balanced flow (Ertel 1942; Hoskins et al. 1985). ${ }^{1}$ It is then possible to "invert" the PV variable to diagnostically recover the balanced components of variables such as the pressure, velocity, and temperature. In this dry atmosphere case, the inversion requires the solution of a linear elliptic partial differential equation (PDE) with suitable boundary conditions once the PV distribution is known.

For moist dynamics including phase changes, one may similarly ask: How can the flow field and variables associated with moisture be decomposed into their balanced and unbalanced components? This is the main topic of this paper. Many important differences arise in the moist case when compared with the dry case, and phase changes create some particularly subtle effects. One of the main objectives of this paper is to describe these differences and subtleties, and to illustrate them using numerical simulations.

A brief overview is as follows. To recover the balanced components of the moist flow, one first must find the relevant low-frequency modes of the system. This is the source of one key difference between the dry and moist cases. In the moist case, the low-frequency component can no longer be described by a single dynamic PV variable; for a moist system, it is necessary to additionally retain a number of dynamically significant moist variables (Smith and Stechmann 2017). Namely, the vortical mode of dry dynamics will be augmented in the moist system with additional low-frequency moist modes. These additional moist modes, which we call $M$ modes or $M$ variables, prove vital in describing the moist balanced flow (Wetzel et al. 2019). In particular, the balanced PV and $M$ variables are both needed together to specify an invertibility principle, which we call $\mathrm{PV}$-and- $M$ inversion, to diagnostically recover balanced components of all other dynamic variables, including moisture. Thus, in analogy to dry dynamics, the balanced flow is obtained from an inversion of balanced PV, although now also with additional balanced moisture components. In practice, the inversion requires the solution of an elliptic PDE with suitable boundary conditions and global knowledge of not only the PV variable but also $M$ variables. In the case with phase changes, the elliptic PDE also now has discontinuous coefficients resulting from phase changes.

\footnotetext{
${ }^{1}$ This may be done more easily with the assumption of small Rossby and Froude numbers, in which case the Ertel PV variable is now approximated by a corresponding quasigeostrophic PV variable, as will be the case throughout this paper.
}

Some prior studies have explored inversion principles to recover the balanced component of a moist system using a single moist PV variable (e.g., Schubert et al. 2001; Marquet 2014). In such cases, some subtleties arise, and we use the present paper to discuss these issues in the context of the more recent concept of $\mathrm{PV}$-and- $M$ inversion. In essence, moist $\mathrm{PV}$ variables generalize the PV of dry dynamics - constructed using the dry-air potential temperature $\theta$, which is inadequate to describe a moist system. Moist PV alternatives have been considered using the virtual potential temperature $\theta_{v}$, the equivalent potential temperature $\theta_{e}$, or some other variable associated with the moist-air entropy. While these moist PV variables have a number of desirable traits from the point of view of moist dynamics and balanced flow, they are not sufficient to individually recover the full moist balanced flow including moisture constituents. For example, it is observed in Schubert et al. (2001) that, using the moist PV defined in terms of $\theta_{v}$, denoted here as $\mathrm{PV}_{v}$, one can define an invertibility principle. However, its inversion recovers only wind and thermal variables of the flow, but not the moisture variables. Similarly, a PV can be defined from $\theta_{e}$ alone (e.g., Bennetts and Hoskins 1979; Emanuel 1979), denoted here by $\mathrm{PV}_{e}$, but it fails to possess an invertibility principle (Cao and Cho 1995; Schubert et al. 2001). In this paper we show, in fact, that $\mathrm{PV}_{v}$ is not balanced and therefore, for a moist system with phase changes, $\mathrm{PV}_{v}$ inversion does not recover the balanced component of the flow. Moreover, $\mathrm{PV}_{e}$ is a suitable $\mathrm{PV}$ variable for $\mathrm{PV}$ and $-M$ inversion and may be used to recover the moist balanced flow. Therefore, the lack of an invertibility principle for $\mathrm{PV}_{e}$ alone highlights the absolute necessity of the balanced $M$ components in the inversion principle.

While some common PV variables, such as $\mathrm{PV}_{v}$, may not be balanced, we also note that they can still be useful quantities for analyzing the atmosphere. For instance, $\mathrm{PV}_{v}$ is conserved for an unsaturated atmosphere, and it changes due to latent heating. Therefore, $\mathrm{PV}_{v}$ or other similar PVs can still be useful quantities for monitoring and diagnosing the effects of latent heating (e.g., Davis and Emanuel 1991; Lackmann 2002; Gao et al. 2004; Brennan and Lackmann 2005; Martin 2006; Brennan et al. 2008; Lackmann 2011; Madonna et al. 2014; Büeler and Pfahl 2017).

The paper is organized as follows. We begin with an illustration of the balanced and unbalanced components of moisture arising from a Boussinesq model in section 2. In particular, we use this model to discuss some of the key features of each component in a simplified setup. In section 3, we introduce the moist anelastic equations to derive evolution equations of PV 
and $M$, discuss PV-and- $M$ inversion with phase changes, and describe how a balanced-unbalanced decomposition may be done in the moist system. We finish the section by highlighting the subtle fact that, since the $\mathrm{PV}-M$ formulation is not unique, some PV choices-such as those found in dry dynamics-may not be balanced for a moist system with phase changes, whereas others indeed lead to equivalent formulations for $\mathrm{PV}$-and- $M$ inversion. In the remaining two sections of the paper we present in more detail the key properties of the $M$ variables by considering solutions of the simplified Boussinesq model and hemispheric-sized simulations using the Weather Research and Forecasting (WRF) Model. In section 4, we discuss that the new moisture $M$ variables hold properties analogous to conserved quantities such as PV variables. In section 5, we highlight key properties of the $M$ variables that distinguish them from thermodynamic variables arising from the moist anelastic system.

\section{Illustration of balanced and unbalanced moisture}

Is moisture a balanced variable, is it an unbalanced variable, or does it have both balanced and unbalanced components? As an initial motivation, we present a numerical simulation that illustrates that moisture has both balanced and unbalanced components.

We simulate a moist Boussinesq fluid with two phases of water-vapor and liquid-in a triply periodic domain. The fluid is rapidly rotating and strongly stratified so that the Rossby and Froude numbers are small (both taken to be 0.1). The model is initialized using a dry turbulent state first generated without the influence of moisture. A large-scale random forcing is then imposed, and the simulation is run to a statistical steady state to provide a dry turbulent state. Moisture in the initial state is then included in a simple way; at a new time $t=0$ a bubble of water vapor is added to the turbulent flow at the center of the domain. The system is then allowed to evolve according to moist Boussinesq dynamics with phase changes of water.

To decompose moisture into balanced and unbalanced components, we use a new type of PV inversion principle, which is described in detail in section 3 and was originally presented in Wetzel et al. (2019). Phase changes are not necessary to show the balanced and unbalanced nature of moisture, but we allow them here for additional realism. The Boussinesq model as given here provides a particularly simple testbed to showcase these features without the undo complexity of additional moisture variables or model parameters. While it is the anelastic equations that are of most interest for atmospheric dynamics, we use a Boussinesq system in this section, with constant buoyancy frequencies, to focus on the basic concepts with this initial illustration. For reference we include the Boussinesq equations in appendix A.

We begin by showing the time evolution of the total water mixing ratio $q_{t}$ and its balanced and unbalanced components in Figs. 1 and 2; the "total" water $q_{t}$ is the sum of the water vapor and liquid water. The model has been advectively nondimensionalized so that 1 time unit corresponds to the time scale associated with balanced motions, whereas a 0.1 time unit is more closely linked to the unbalanced (or fast) motions.

Figure 1 shows the decomposition of $q_{t}$ into two components. The decomposition is not obtained from time averaging but rather through a type of moist PV inversion that is described in subsequent sections. In particular, the balanced and unbalanced components are calculated at each time step from the available variables at that time step (i.e., they are calculated diagnostically). Nevertheless, while no time averaging was used in their creation, the two components appear to identify distinctly different time evolutions that describe the slowly and rapidly evolving parts of $q_{t}$; and they are therefore accordingly named the balanced and unbalanced components, respectively.

Moreover, in Figs. 1 and 2, it is seen that the balanced component of $q_{t}$ closely tracks the broad features or large-scale structure of the initial water bubble. Beyond that, the unbalanced component can also be seen to contribute additional details, on both the short and long time scales. Therefore, the moisture is principally balanced with the unbalanced component adding significant small-scale structure to the overall moisture variable.

\section{PV inversion for a class of moist PV definitions}

How can PV inversion be carried out for a moist system? It is known (see, e.g., Cao and Cho 1995; Schubert et al. 2001), that PV inversion in the traditional sense cannot be performed if the moist PV is defined based on equivalent potential temperature, $\theta_{e}$. Here we will show that, in fact, one can do a type of inversion with many definitions of PV, including a PV based on $\theta_{e}$. Rather than traditional PV inversion, it is actually PV-and- $M$ inversion, accounting for the additional balanced components $M$ of a moist system.

Furthermore, while we show that many PV definitions will suffice, we also show that some common PV definitions are not balanced. In particular, the PV defined 

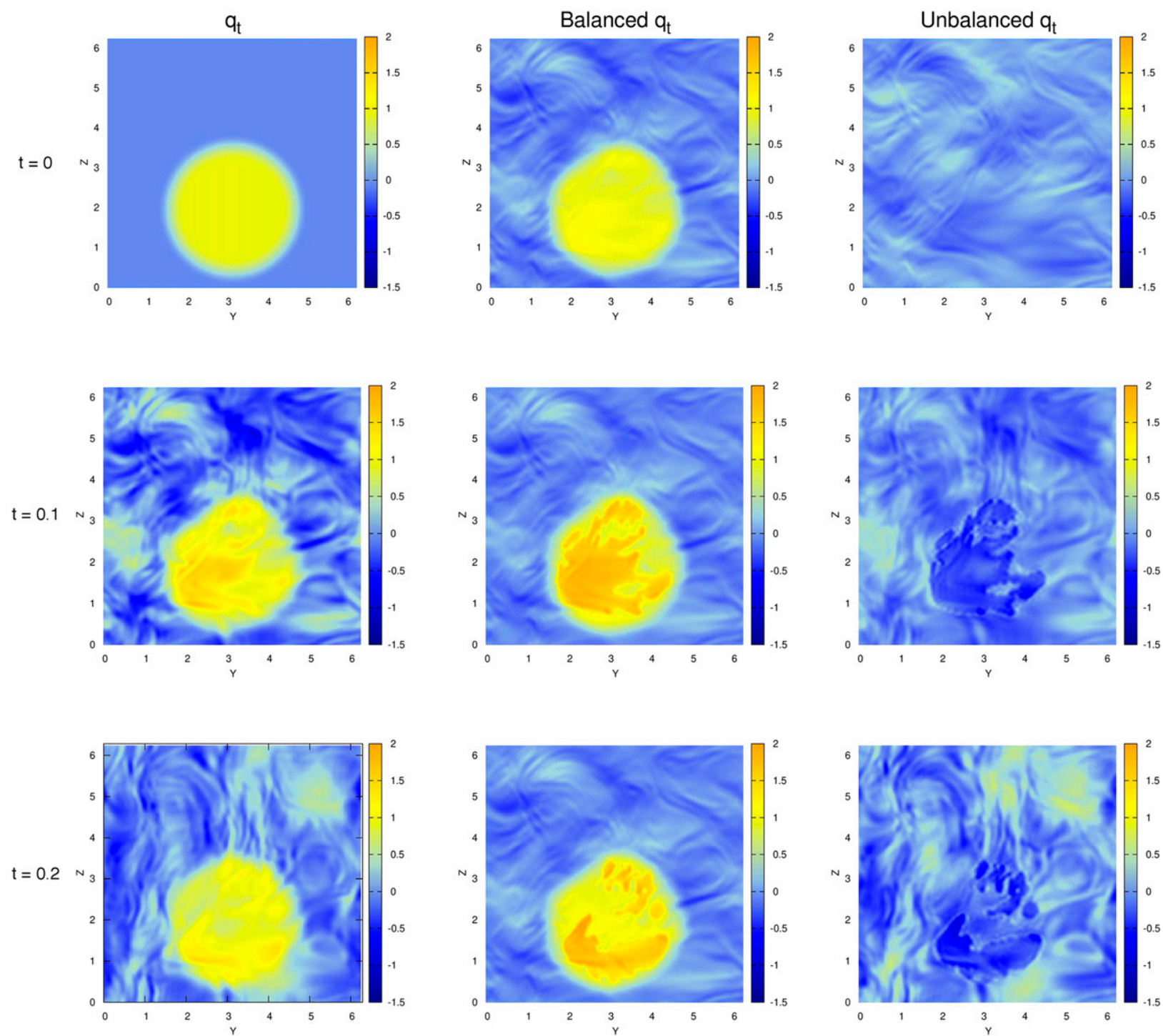

FIG. 1. Evolution of (left) total water $q_{t}$ and its (center) balanced and (right) unbalanced components over a "short" time (top) $t=0$, (middle) $t=0.1$, and (bottom) $t=0.2$. A slice is shown of each of the $3 \mathrm{D}$ variables; e.g., $q_{t}(\pi, y, z)$ is plotted with $x=\pi$ held fixed.

using potential temperature $\theta\left(\mathrm{PV}_{\theta}\right)$, and the $\mathrm{PV}$ based on virtual potential temperature $\theta_{v}\left(\mathrm{PV}_{v}\right)$ are not balanced. Therefore, an inversion based on either of these PVs does not extract the balanced component of a moist system with phase changes.

\section{a. Anelastic equations with warm-rain microphysics}

In this section, we describe the moist system that will be used throughout the paper. It is the anelastic equations of motion for a moist atmosphere containing three moist variables: water vapor, cloud water, and rainwater (e.g., Lipps and Hemler 1982; Grabowski and Smolarkiewicz 1996; Hernández-Dueñas et al. 2013;
Klein and Majda 2006). The system may be written in the following form:

$$
\begin{aligned}
\frac{D \mathbf{u}}{D t}+f \hat{\mathbf{z}} \times \mathbf{u} & =-\nabla\left(\frac{p}{\tilde{\rho}}\right)+\hat{\mathbf{z}} b, \\
\nabla \cdot(\tilde{\rho} \mathbf{u}) & =0, \\
\frac{D \theta_{e}}{D t}+w \frac{d \tilde{\theta}_{e}}{d z} & =0, \\
\frac{D q_{t}}{D t}+w \frac{d \tilde{q}_{t}}{d z} & =\frac{1}{\tilde{\rho}} \frac{\partial}{\partial z}\left(\tilde{\rho} V_{T} q_{r}\right), \quad \text { and }
\end{aligned}
$$



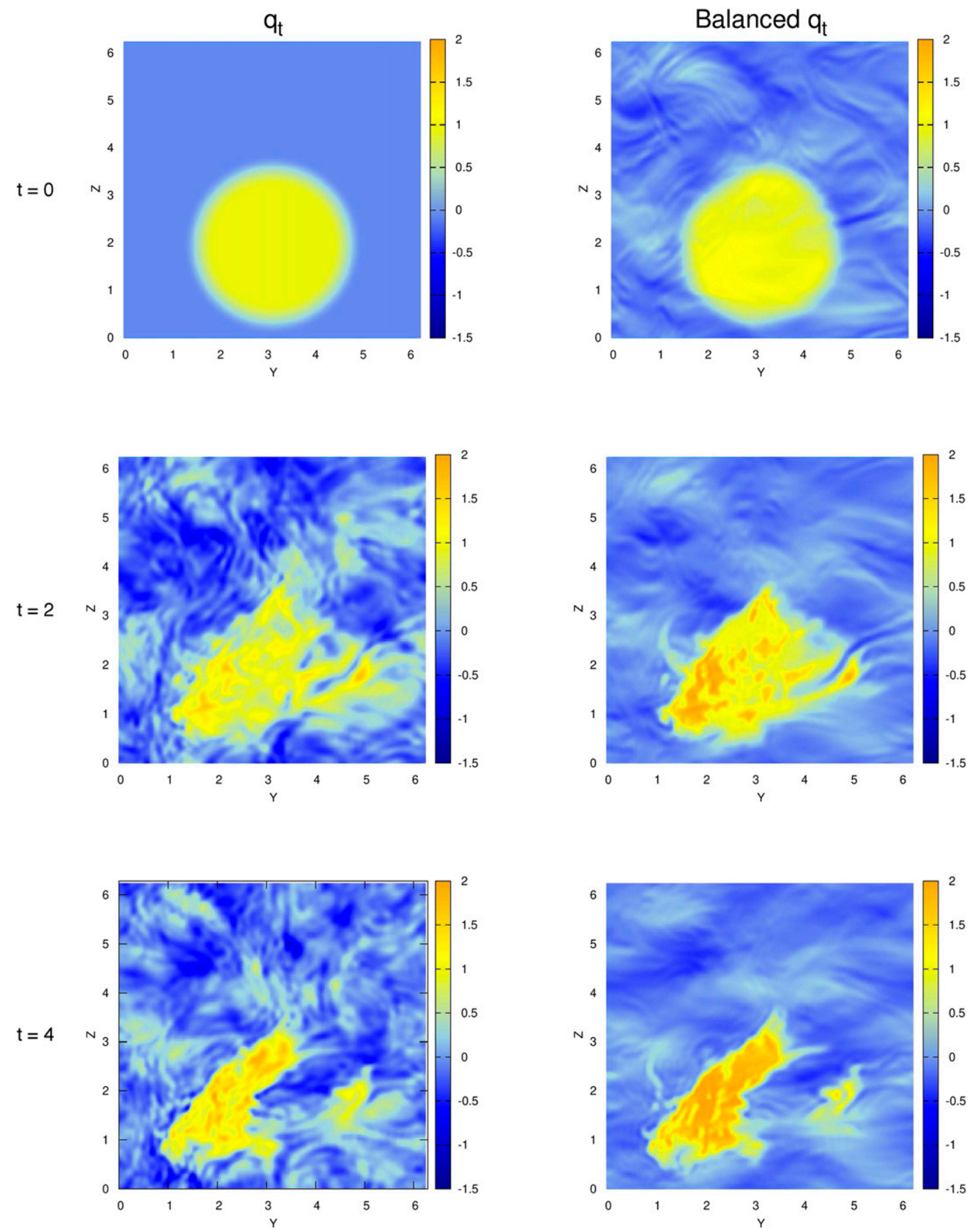

FIG. 2. Similar to Fig. 1, but the evolution is shown over a "long" time (top) $t=0$, (middle) $t=2$, and (bottom) $t=4$.

$$
\frac{D q_{r}}{D t}=\frac{1}{\tilde{\rho}} \frac{\partial}{\partial z}\left(\tilde{\rho} V_{T} q_{r}\right)+A_{r}+C_{r}-E_{r} .
$$

The variables in the system of equations are as follows: $\rho$ is the density; $p$ is the pressure; $\mathbf{u}$ is the velocity with Cartesian components $(u, v, w)$, where $u$ is the zonal (westeast) speed, $v$ is the meridional (south-north) speed, and $w$ is the vertical (down-up) speed; $q_{t}$ is the total water mixing ratio, defined as the sum of all three moisture components:

$$
q_{t}=q_{v}+q_{c}+q_{r}
$$

where $q_{v}$ is the water vapor mixing ratio, $q_{c}$ is the cloud water mixing ratio, and $q_{r}$ is the rainwater mixing ratio; 
$\theta_{e}$ is the equivalent potential temperature, defined in linearized form $^{2}$ in terms of the potential temperature $\theta$ and water vapor $q_{v}$ as

$$
\theta_{e}=\theta+\tilde{\gamma} q_{v},
$$

where $\tilde{\gamma}=L_{v} /\left(c_{p} \tilde{\Pi}\right)$ with $\tilde{\Pi}=\tilde{T} / \tilde{\theta}=\left(\tilde{p} / p_{0}\right)^{R_{d} / c_{p}}$ being the Exner function for nondimensionalized pressure $\left(p_{0}\right.$ is the reference surface pressure and $T$ is the temperature); and $b$ is the buoyancy, defined by the linear combination

$$
b=g\left(\frac{\theta}{\tilde{\theta}}+\varepsilon_{0} q_{v}-q_{c}-q_{r}\right) .
$$

In addition, the following parameters are used: the acceleration due to gravity $g$, the Coriolis parameter $f$, the latent heat of vaporization $L_{v}$, the specific heat at constant pressure for dry air $c_{p}$, the ratio of water vapor $R_{v}$ and dry air $R_{d}$ gas constants $\varepsilon_{0}=\left(R_{v} / R_{d}\right)-1$, and the terminal speed of falling rain drops $V_{T}$. Here, the operator $D / D t=$ $\partial / \partial t+\mathbf{u} \cdot \nabla$ denotes the three-dimensional material derivative with gradient $\nabla=(\partial / \partial x, \partial / \partial y, \partial / \partial z)$ and $\hat{\mathbf{z}}=\nabla z$ is the unit vector in the vertical direction.

The thermodynamic variables $\rho, p, \theta_{e}$ and moisture variables $q_{t}, q_{v}, q_{c}$, and $q_{r}$ have been decomposed into anelastic background states, denoted by $\widetilde{()}$, and their respective anomalies. The background states are taken to be profiles of only the height $z$ such that the density and pressure are hydrostatically balanced,

$$
\begin{aligned}
\tilde{q}_{c} & =\tilde{q}_{r}=0 \quad \text { so that } \quad \tilde{q}_{t}=\tilde{q}_{v} \quad \text { and } \\
\frac{d \tilde{\theta}_{e}}{d z} & =\frac{d \tilde{\theta}}{d z}+\tilde{\gamma} \frac{d \tilde{q}_{v}}{d z} .
\end{aligned}
$$

The Exner function $\tilde{\Pi}$ and the coefficient $\tilde{\gamma}$ are thus functions of $z$ only. The anomalies, in turn, are functions of the three-dimensional position $(x, y, z)$ and time $t$. So, for example, the equivalent potential temperature is decomposed into an anelastic background $\tilde{\theta}_{e}(z)$ and perturbation $\theta_{e}(x, y, z, t)$.

The source terms in (1e) correspond to the autoconversion of cloud water into rainwater $A_{r}$, the collection of cloud water to form rainwater $C_{r}$, and the evaporation of rainwater into water vapor $E_{r}$. The source terms require microphysics modeling that is beyond the scope of this paper, but they may be considered as nonlinear functions of the three moisture phases $q_{v}, q_{c}$, and $q_{r}$ and the height $z$;

\footnotetext{
${ }^{2}$ Note that the linearized form of $\theta_{e}$ is used in (2b) for simplicity, because it allows for explicit analytical expressions in the equations of PV-and- $M$ inversion in, for example, (8) and (21b). More complex expressions for $\theta_{e}$ (e.g., Emanuel 1994; Stevens 2005) could potentially be used but would lead to more complicated formulas.
}

we refer the reader interested in the particulars of these source terms in the case of the Kessler parameterization to, for example, Kessler (1969) and Grabowski and Smolarkiewicz (1996).

The moisture constituents are constrained so that cloud water $q_{c}$ is not present in unsaturated regions and water vapor $q_{v}$ does not exceed its saturation value in saturated regions (Grabowski and Smolarkiewicz 1996). Namely, the moisture variables satisfy the constraints

$$
\begin{array}{lll}
q_{v}<q_{v s}, & q_{c}=0 & \text { (unsaturated) } \\
q_{v}=q_{v s}, & q_{c} \geq 0 & \text { (saturated), }
\end{array}
$$

where $q_{v s}$ is the saturation water vapor that, for simplicity, is assumed to be a known profile of $z$. Since no constraints are applied to the rainwater (aside from $q_{r} \geq$ 0 ), we allow the existence of rainwater $q_{r}$ in both unsaturated and saturated regions. Similarly, using definition (2a), we may note that constraints (4a) and (4b) may be written in the form

$$
\begin{aligned}
& q_{t}-q_{r}<q_{v s}, \quad q_{c}=0 \quad \text { (unsaturated) and } \\
& q_{t}-q_{r} \geq q_{v s}, \quad q_{v}=q_{v s} \quad \text { (saturated) }
\end{aligned}
$$

Therefore, the total water $q_{t}$ and the rainwater $q_{r}$ are sufficient to determine the location of unsaturated and saturated regions and allow us to define the indicator functions for unsaturated and saturated regions to be

$$
H_{u}=\left\{\begin{array}{lll}
1 & \text { for } & q_{t}-q_{r}<q_{v s} \\
0 & \text { for } & q_{t}-q_{r} \geq q_{v s}
\end{array} \text { and } H_{s}=1-H_{u},\right.
$$

respectively. Indeed, it follows that it is enough to know $q_{t}$ and $q_{r}$ to determine all moisture phases; both water vapor $q_{v}$ and cloud water $q_{c}$ may be determined diagnostically using

$$
\begin{aligned}
& q_{v}=\min \left(q_{t}-q_{r}, q_{v s}\right) \quad \text { or } \\
& q_{v}=\left(q_{t}-q_{r}\right) H_{u}+q_{v s} H_{s} \text { and } \\
& q_{c}=\max \left(0, q_{t}-q_{r}-q_{v s}\right) \quad \text { or } \quad q_{c}=\left(q_{t}-q_{r}-q_{v s}\right) H_{s} .
\end{aligned}
$$

Because of these moisture constraints, it is possible to write the buoyancy $b$ purely in terms of the dynamic variables $\theta_{e}, q_{t}$, and $q_{r}$. To accomplish this, it is convenient to consider the buoyancy in the unsaturated and saturated regions separately. Namely, the buoyancy may be written as

$$
b=b_{u} H_{u}+b_{s} H_{s},
$$


where $b_{u}$ and $b_{s}$ are the buoyancy in the unsaturated and saturated regions, respectively. In each region, we may use (2a)-(2b) and (5a)-(5b) on buoyancy (2c) to obtain

$$
\begin{aligned}
& b_{u}=g\left[\frac{\theta_{e}}{\tilde{\theta}}+\left(\varepsilon_{0}+1-\frac{\tilde{\gamma}}{\tilde{\theta}}\right)\left(q_{t}-q_{r}\right)-q_{t}\right] \text { and } \\
& b_{s}=g\left[\frac{\theta_{e}}{\tilde{\theta}}+\left(\varepsilon_{0}+1-\frac{\tilde{\gamma}}{\tilde{\theta}}\right) q_{v s}-q_{t}\right]
\end{aligned}
$$

as explicit expressions for defining $b_{u}$ and $b_{s}$ in terms of $\theta_{e}$ and $q_{t}$.

\section{b. Leading-order balance conditions}

Our goal is to define the balanced and unbalanced components of the moist system, and therefore the balance conditions must be defined. In analogy to the dry case, the quasigeostrophic (QG) setting of small Rossby and Froude numbers is used, and the leadingorder balance conditions are geostrophic balance,

$$
f u=-\frac{\partial}{\partial y}\left(\frac{p}{\tilde{\rho}}\right) \text { and } f v=\frac{\partial}{\partial x}\left(\frac{p}{\tilde{\rho}}\right)
$$

and hydrostatic balance,

$$
b=\frac{\partial}{\partial z}\left(\frac{p}{\tilde{\rho}}\right) .
$$

Further details, which are omitted here for the sake of brevity, are described by Smith and Stechmann (2017) and Wetzel et al. (2019). One important point to note, however, is the difference between the dry case and the moist case: in the moist case, the buoyancy in (9b) will take a different form in unsaturated and saturated regions, as shown in (8).

Furthermore, the buoyancy at leading order will take a simplified form. In particular, (2c) becomes $b=$ $(g \theta / \tilde{\theta})+O(\mathrm{Ro})$ for small Rossby number Ro since $c_{p} \tilde{\theta}(0) / L_{v} \approx 0.1$ is small. Thus, explicit contributions from the moisture terms $q_{v}, q_{c}$, and $q_{r}$ vanish and the buoyancy is directly proportional to the potential temperature at leading order:

$$
b=g \frac{\theta}{\tilde{\theta}} .
$$

This means that, at leading order, $(8 \mathrm{a})-(8 \mathrm{c})$ relate the unsaturated buoyancy $b_{u}$ and saturated buoyancy $b_{s}$ with $\theta_{e}$ and $q_{t}$ as

$$
\begin{aligned}
b_{u} & =\frac{g}{\tilde{\theta}}\left[\theta_{e}-\tilde{\gamma}\left(q_{t}-q_{r}\right)\right] \text { and } \\
b_{s} & =\frac{g}{\tilde{\theta}}\left(\theta_{e}-\tilde{\gamma} q_{v s}\right) .
\end{aligned}
$$

In terms of the buoyancy $b$ we have

$$
b=\frac{g}{\tilde{\theta}}\left[\theta_{e}-\tilde{\gamma}\left(q_{t}-q_{r}\right) H_{u}-\tilde{\gamma} q_{v s} H_{s}\right]
$$

as a simplified, leading-order version of (8).

\section{c. Definition of classes of $P V$ and $M$ variables}

Here, we describe the potential vorticity (PV) and moisture $(M)$ variables that characterize the balanced components of the system. Two main points are emphasized. First, in the moist case, the PV variable alone is not sufficient to characterize the balanced part of the system; additional moisture $(M)$ variables are needed. Second, many definitions of the PV variable are possible, and we show how to construct a class of suitable PV definitions.

To describe the evolution of the balanced part of the anelastic equations [(1a)-(1e)], the next-to-leading-order terms are considered, and they take the form

$$
\begin{gathered}
\frac{D_{H} \zeta}{D t}=\frac{f}{\tilde{\rho}} \frac{\partial}{\partial z}(\tilde{\rho} w)+O(\mathrm{Ro}), \\
\frac{D_{H} \theta_{e}}{D t}+w \frac{d \tilde{\theta}_{e}}{d z}=O(\mathrm{Ro}), \\
\frac{D_{H} q_{t}}{D t}+w \frac{d \tilde{q}_{t}}{d z}=\frac{1}{\tilde{\rho}} \frac{\partial}{\partial z}\left(\tilde{\rho} V_{T} q_{r}\right)+O(\mathrm{Ro}), \quad \text { and } \\
\frac{D_{H} q_{r}}{D t}=\frac{1}{\tilde{\rho}} \frac{\partial}{\partial z}\left(\tilde{\rho} V_{T} q_{r}\right)+A_{r}+C_{r}-E_{r}+O(\mathrm{Ro})
\end{gathered}
$$

as Ro $\rightarrow 0$, where $D_{H} / D t=\partial / \partial t+\mathbf{u}_{H} \cdot \nabla_{H}$ is the horizontal material derivative, and $\zeta=(\nabla \times \mathbf{u}) \cdot \hat{\mathbf{z}}=\partial v / \partial x-\partial u / \partial y$ is the vertical component of the relative vorticity.

The PV and $M$ variables can be defined, on the basis of (12), in many different ways. In principle, we wish to define variables whose evolution equations lack a $w$ term by taking linear combinations of (12a)-(12d). Many different linear combinations are possible, and each leads to a different set of PV and $M$ variables. Next, we illustrate two such possibilities.

As a first possibility, one could consider a PV variable $\mathrm{PV}_{e}$ based on equivalent potential temperature, $\theta_{e}$. The three conserved variables $\mathrm{PV}_{e}, M$, and $M_{r}$ could then be defined as

$$
\begin{aligned}
\mathrm{PV}_{e} & =\zeta+\frac{f}{\tilde{\rho}} \frac{\partial}{\partial z}\left(\frac{\tilde{\rho}}{d \tilde{\theta}_{e} / d z} \theta_{e}\right), \\
M & =q_{t}+\tilde{G}_{M} \theta_{e}, \quad \text { and } \\
M_{r} & =M-q_{r},
\end{aligned}
$$

with evolution equations 


$$
\begin{aligned}
\frac{D_{H} \mathrm{PV}_{e}}{D t} & =-\frac{f}{d \tilde{\theta}_{e} / d z} \frac{\partial \mathbf{u}_{H}}{\partial z} \cdot \nabla_{H} \theta_{e}, \\
\frac{D_{H} M}{D t} & =\frac{1}{\tilde{\rho}} \frac{\partial}{\partial z}\left(\tilde{\rho} V_{T} q_{r}\right), \quad \text { and } \\
\frac{D_{H} M_{r}}{D t} & =E_{r}-A_{r}-C_{r},
\end{aligned}
$$

where $\tilde{G}_{M}=-\left(d \tilde{q}_{t} / d z\right) /\left(d \tilde{\theta}_{e} / d z\right)$ is a ratio of background gradients and is a function of $z$ only. Similar types of $M$ variables have also been considered for other moist systems (e.g., Frierson et al. 2004; Stechmann and Majda 2006; Chen and Stechmann 2016).

The variables $\mathrm{PV}_{e}, M$, and $M_{r}$ represent the evolution of the balanced moist flow or the slow dynamics of the moist anelastic system. The PV and $M$ variables are balanced in the sense that they are all zero-frequency eigenmodes [i.e., if the system (14) is linearized about a resting base state with $\mathbf{u}_{H}=0$, neglecting $V_{T}$ and microphysical source terms, the three eigenvalues are all equal to zero]. Indeed, since the evolution equations of the PV and $M$ variables are formed by eliminating $w$, system (14) is decoupled from waves.

In making an association between the vertical velocity $w$ and waves, a note is needed with regard to the difference between two distinct settings: the balanced setting of low-frequency motions and the unbalanced setting contained in the full atmospheric dynamics. Either setting can be considered under conditions of low Froude number and low Rossby number. The difference between the two settings is in the presence or absence of waves: waves are not present in the balanced setting, while waves are present in the unbalanced setting. In the balanced setting, the dynamics of the system are governed by the QG equations, as indicated by (9)-(12). In strict terms, the $w$ that appears in (12) would not be associated with waves, since the balance conditions in (9) hold under the assumption that $w$ is weak and waves are not present, to leading order. Nevertheless, the terms in (12) do still indicate the association of $w$ and waves that would be found if one were to analyze the equations that include not only balanced but also unbalanced (wave) components. In the unbalanced setting, one can associate $w$ with waves and the association is the same as in (12). For simplicity of exposition, we make the $w$-and-wave association here, without going into details of this unbalanced case. ${ }^{3}$ This note is also relevant to the broader scope of this paper, in the sense that

\footnotetext{
${ }^{3}$ Three of the authors (Smith, Stechmann, and Zhang) have analyzed the equations in the unbalanced case, and these results will be presented elsewhere in the near future.
}

the balanced QG setting in (9)-(12) is used to motivate the ideas of $\mathrm{PV}$-and- $M$ inversion, but the main aim throughout this paper is to analyze the full atmospheric dynamics including unbalanced waves and to separate the balanced and unbalanced components from each other.

As a second possibility (among many) for defining PV and $M$ variables, one could define a $\mathrm{PV}$ variable $\mathrm{PV}_{u}$ based on the unsaturated buoyancy variable, $b_{u}$. To do this, rather than using (12b)-(12c), we may consider the linear combinations that give rise to the unsaturated and saturated buoyancies (11a)-(11b) and lead to the evolution equations

$$
\begin{aligned}
\frac{D_{H} b_{u}}{D t}+N_{u}^{2} w & =\frac{g \tilde{\gamma}}{\tilde{\theta}}\left(A_{r}+C_{r}-E_{r}\right) \quad \text { and } \\
\frac{D_{H} b_{s}}{D t}+N_{s}^{2} w & =0
\end{aligned}
$$

where

$$
N_{u}^{2}=\frac{g}{\tilde{\theta}} \frac{d \tilde{\theta}}{d z} \quad \text { and } \quad N_{s}^{2}=\frac{g}{\tilde{\theta}} \frac{d \tilde{\theta}_{e}}{d z}
$$

are the unsaturated and saturated buoyancy frequencies, respectively. Buoyancy frequencies $N_{u}^{2}$ and $N_{s}^{2}$ are the simplified forms that arise in the small Rossby limit; for more general forms, we refer the reader to, for example, Emanuel (1994), Smith and Stechmann (2017), and Durran and Klemp (1982). Then, (15a) and (15b) may be combined with (12a) and (12d) to obtain the conserved variables

$$
\begin{aligned}
\mathrm{PV}_{u} & =\zeta+\frac{f}{\tilde{\rho}} \frac{\partial}{\partial z}\left(\frac{\tilde{\rho}}{N_{u}^{2}} b_{u}\right), \\
M_{b} & =\frac{b_{s}}{N_{s}^{2}}-\frac{b_{u}}{N_{u}^{2}}, \quad \text { and } \\
M_{q} & =M_{b}+\frac{1}{N_{u}^{2}} \frac{g \tilde{\gamma}}{\tilde{\theta}} q_{r},
\end{aligned}
$$

with evolution equations

$$
\begin{aligned}
\frac{D_{H} \mathrm{PV}_{u}}{D t}= & -\frac{f}{N_{u}^{2}} \frac{\partial \mathbf{u}_{H}}{\partial z} \cdot \nabla_{H} b_{u} \\
& +\frac{f}{\tilde{\rho}} \frac{\partial}{\partial z}\left[\tilde{\rho} \frac{1}{N_{u}^{2}} \frac{g \tilde{\gamma}}{\tilde{\theta}}\left(A_{r}+C_{r}-E_{r}\right)\right], \\
\frac{D_{H} M_{b}}{D t}= & \frac{1}{N_{u}^{2}} \frac{g \tilde{\gamma}}{\tilde{\theta}}\left(E_{r}-A_{r}-C_{r}\right), \text { and } \\
\frac{D_{H} M_{q}}{D t}= & \frac{1}{N_{u}^{2}} \frac{g \tilde{\gamma}}{\tilde{\theta}} \frac{\partial}{\tilde{\rho}} \frac{\partial}{\partial z}\left(\tilde{\rho} V_{T} q_{r}\right) .
\end{aligned}
$$


This set of variables $\mathrm{PV}_{u}, M_{b}$, and $M_{q}$ provides another characterization of the balanced component of the system, in addition to the example of $\mathrm{PV}_{e}, M$, and $M_{r}$ described in (13).

Many other definitions of $\mathrm{PV}$ and $M$ variables are possible. In broad terms, any linear combination of the equivalent potential temperature (12b) and total water (12c) may be used to eliminate the $w$ term in the relative vorticity equation given in (12a). This class of linear combinations defines a class of PV variables. Similarly, a class of $M$ variables is defined by the linear combinations of $M$ in (13b), $M_{r}$ in (13c), and $q_{v s}$.

\section{d. $P V$-and- $M$ inversion}

We now describe how knowledge of $\mathrm{PV}_{e}, M$, and $M_{r}$ may be used to recover the balanced streamfunction $\psi$. In the dry case, this process is called PV inversion, and only the PV variable is needed. In the moist case, in contrast, the moist $M$ variables are also needed, and we therefore use the term PV-and- $M$ inversion. The balanced streamfunction $\psi$ and the $\mathrm{PV}-M$ variables may then be used to determine the balanced components of all flow variables; the special case of recovering the balanced moisture is discussed in appendix B.

From the balance conditions described in section $3 b$, one can see that a streamfunction $\psi$ can be defined in terms of the pressure as $\psi=p /(f \tilde{\rho})$, and the balance conditions can be written in terms of $\psi$ as

$$
\begin{aligned}
\mathbf{u}_{H} & =\left(-\frac{\partial \psi}{\partial y}, \frac{\partial \psi}{\partial x}\right) \quad \text { and } \\
b & =f \frac{\partial \psi}{\partial z} .
\end{aligned}
$$

These balance conditions are essentially the same for a dry or moist system, aside from the important difference that buoyancy $b$ can change form as a result of phase changes of water.

To define an elliptic PDE for PV-and- $M$ inversion, the starting point is the definition of $\mathrm{PV}_{e}$, from (13a), which we rewrite here again for convenience:

$$
\mathrm{PV}_{e}=\zeta+\frac{f}{\tilde{\rho}} \frac{\partial}{\partial z}\left(\frac{\tilde{\rho}}{d \tilde{\theta} e_{e} / d z} \theta_{e}\right)
$$

This $\mathrm{PV}_{e}$ definition can then be turned into an elliptic PDE by writing $\zeta$ and $\theta_{e}$ in terms of the variables $\psi, M$, $M_{r}$, and $z$. First, the relative vorticity $\zeta$ is directly related to only the streamfunction via

$$
\zeta=\nabla_{H}^{2} \psi
$$

Second, the equivalent potential temperature $\theta_{e}$ may be written in terms of $\psi, M, M_{r}$, and $z$ by solving for $\theta_{e}$ in (11c) and using the buoyancy equations in (11c) and (18b) and the definitions (13b) and (13c). That is, we may use (13b) and (13c) on (11c) to obtain

$$
b=\frac{g}{\tilde{\theta}}\left[\theta_{e}-\tilde{\gamma}\left(M_{r}-\tilde{G}_{M} \theta_{e}\right) H_{u}-\tilde{\gamma} q_{v s} H_{s}\right] .
$$

Next, using the fact that $b=g \theta / \tilde{\theta}$ as in (10) and solving for $\theta_{e}$ we obtain

$$
\frac{1}{d \tilde{\theta}_{e} / d z} \theta_{e}=\frac{1}{d \tilde{\theta} / d z}\left(\theta+\tilde{\gamma} M_{r}\right) H_{u}+\frac{1}{d \tilde{\theta}_{e} / d z}\left(\theta+\tilde{\gamma} q_{v s}\right) H_{s} .
$$

Last, inserting (20) and (21b) into the definition of $\mathrm{PV}_{e}$ in (13a), we arrive at

$$
\begin{aligned}
\nabla_{H}^{2} \psi & +\frac{1}{\tilde{\rho}} \frac{\partial}{\partial z}\left[\tilde{\rho} \frac{f^{2}}{N_{u}^{2}}\left(\frac{\partial \psi}{\partial z}+\frac{g \tilde{\gamma}}{f \tilde{\theta}} M_{r}\right) H_{u}\right. \\
+\tilde{\rho} & \left.\frac{f^{2}}{N_{s}^{2}}\left(\frac{\partial \psi}{\partial z}+\frac{g \tilde{\gamma}}{f \tilde{\theta}} q_{v s}\right) H_{s}\right]=\mathrm{PV}_{e}
\end{aligned}
$$

which is an elliptic PDE for $\psi$.

For some intuition on the derivation of (22), note that the basic principle was simply a transformation between different thermodynamic variables. Specifically, (21) was used to write $\theta_{e}$ in terms of $\partial \psi / \partial z, M, M_{r}$, and $z$, and these four variables were chosen because they define the balanced component of the thermodynamic part of the system. To see this, note that $\partial \psi / \partial z$ is the balanced part of $\theta$, and $M$ and $M_{r}$ are themselves balanced variables, and $z$ plays the role of pressure for an anelastic system (e.g., Pauluis 2008) since $p \approx \tilde{p}(z)$. Hence, $\partial \psi / \partial z, M$, $M_{r}$, and $z$ can be viewed as the balanced component of $\theta, M, M_{r}$, and $p$, which are a different set of four thermodynamic variables, other than the original four $\theta_{e}, q_{t}, q_{r}$, and $p$ that were used to formulate the anelastic system (1).

The inversion PDE (22) could be considered either to be linear or nonlinear (as a function of the streamfunction $\psi$ ), depending on assumptions. In a purely balanced setting, as for the QG equations (Smith and Stechmann 2017), the inversion PDE (22) is nonlinear in the streamfunction $\psi$. This is because the Heaviside functions, $H_{u}$ and $H_{s}$, depend on the total water $q_{t}$, which itself is a function of the streamfunction $\psi\left(\right.$ and $M_{r}$ ). On the other hand, in a mixed setting with both balanced and unbalanced components present, the inversion PDE (22) could be treated as being linear in the streamfunction $\psi$. In this case, the Heaviside functions, $H_{u}$ and $H_{s}$, are taken to be known functions that are given by the 
available data. In this sense, the given information includes the PV and $M$ variables, the boundary conditions, and the phase interface locations (i.e., the Heaviside functions $H_{u}$ and $H_{s}$ ). This will be the scenario used in this paper, since the aim is to analyze data of atmospheric dynamics, including not only balanced but also unbalanced components.

\section{e. Equivalence of many different $P V$-and- $M$ inversions}

Many different choices of $\mathrm{PV}-M$ variables are suitable to recover the balanced flow of the system. That is, though different versions of $\mathrm{PV}-M$ variables may be constructed, they will all recover the same balanced streamfunction, so long as they are derived by eliminating $w$ from the system (12).

As an example for illustration, we show the equivalence between two different $\mathrm{PV}$-and- $M$ inversions: the $\mathrm{PV}$-and- $M$ inversion using $\mathrm{PV}_{e}$ in system (13), and the $\mathrm{PV}$-and- $M$ inversion using $\mathrm{PV}_{u}$ in system (16). The inversion for $\mathrm{PV}_{e}$ was derived earlier in (22), and the inversion for $\mathrm{PV}_{u}$ can be derived as follows. The starting point is the $\mathrm{PV}_{u}$ definition in (16a). To turn this $\mathrm{PV}_{u}$ definition into an elliptic PDE for the streamfunction, we first write $b_{u}$ in terms of $b, M_{b}$, and $M_{q}$ by using (16b) on $(8 \mathrm{a})$. This gives the equation

$$
\frac{1}{N_{u}^{2}} b_{u}=\frac{1}{N_{u}^{2}} b H_{u}+\left(\frac{1}{N_{s}^{2}} b-M_{b}\right) H_{s} .
$$

Then, substituting (20), (23), and (18b) into (16a), we arrive at the inversion principle involving $\mathrm{PV}_{u}$ :

$$
\nabla_{H}^{2} \psi+\frac{1}{\tilde{\rho}} \frac{\partial}{\partial z}\left[\tilde{\rho} \frac{f^{2}}{N_{u}^{2}} \frac{\partial \psi}{\partial z} H_{u}+\tilde{\rho}\left(\frac{f^{2}}{N_{s}^{2}} \frac{\partial \psi}{\partial z}-f M_{b}\right) H_{s}\right]=\mathrm{PV}_{u} .
$$

This defines a second variant of $\mathrm{PV}$-and- $M$ inversion, in addition to the earlier case involving $\mathrm{PV}_{e}$ in (22).

The equivalence of the two PV-and- $M$ inversions (22) and (24) is due to the fact that they recover the same streamfunction when identical boundary conditions are used. To show this, we take the difference between the inversion (22) for streamfunction $\psi_{e}$ and the inversion (24) for streamfunction $\psi_{u}$. The result is

$$
\mathscr{C}\left(\psi_{e}-\psi_{u}\right)=0,
$$

where the differential operator $\mathscr{A}$ is defined as

$$
\mathscr{C}=\nabla_{H}^{2}+\frac{1}{\tilde{\rho}} \frac{\partial}{\partial z}\left(\tilde{\rho} \frac{f^{2}}{N_{u}^{2}} H_{u} \frac{\partial}{\partial z}+\tilde{\rho} \frac{f^{2}}{N_{s}^{2}} H_{s} \frac{\partial}{\partial z}\right) ;
$$

see appendix $\mathrm{C}$ for details on the derivation of (25). Equation (25) is a PDE for the difference $\psi_{e}-\psi_{u}$ of the streamfunctions. Note that the PDE in (25) is homogeneous (i.e., the right-hand side is zero), and the boundary conditions for the difference $\psi_{e}-\psi_{u}$ are also homogeneous (i.e., zero). Therefore, the solution to (25) is $\psi_{e}-\psi_{u}=0$, so the streamfunctions must equal each other over that domain: $\psi_{e}=\psi_{u}$. Therefore, the $P V$-and- $M$ inversions in (22) and (24) recover identical streamfunctions.

Indeed, any PV and $M$ variables of the class obtained from linear combinations of (12) to remove the $w$ terms will lead to PV-and- $M$ inversions that recover the balanced streamfunction. This may be principally understood by the fact that these PV- $M$ variables will have no background state and, therefore, their evolution is not directly affected by fast waves.

From another perspective, if one views (9)-(12) as a quasigeostrophic theory in a purely balanced setting, then it is clear from the asymptotic derivation that different choices of the $\mathrm{PV}$ and $M$ variables are all associated with the same flow. This is because the fundamental QG equations are given by (12) and any reshuffling of the equations (to define different PV and $M$ variables) will describe the same QG dynamics and the same flow. On the other hand, if we consider the setting of full atmospheric dynamics, including not only balanced but also unbalanced components, then some definitions of PV and $M$ variables may not be suitable to recover the balanced component of the flow; in this setting, which is the main setting of this paper, it is important to understand if a PV variable is balanced or if, instead, it is influenced by the vertical velocity $w$ and waves.

\section{f. Some common PVs are not balanced}

Interestingly, not all choices of PV will lead to an inversion principle that recovers the balanced streamfunction. We illustrate this point by considering the PV defined in terms of virtual potential temperature $\theta_{v}$. We define this PV variable as

$$
\mathrm{PV}_{v}=\zeta+\frac{f}{\tilde{\rho}} \frac{\partial}{\partial z}\left(\frac{\tilde{\rho}}{N_{u}^{2}} b\right)
$$

using $b$, since, in the limit of small Froude and Rossby numbers, the virtual potential temperature $\theta_{v}$ is proportional to the potential temperature $\theta$ or the buoyancy $b$. The variable $\mathrm{PV}_{v}$ is a linearized version of the moist PV used by Schubert et al. (2001) and is a natural PV to consider in a moist system. An inversion principle directly follows from inserting $\zeta=\nabla_{H}^{2} \psi$ and $b=f \partial \psi / \partial z$ into (26), which leads to 


$$
\nabla_{H}^{2} \psi+\frac{1}{\tilde{\rho}} \frac{\partial}{\partial z}\left(\tilde{\rho} \frac{f^{2}}{N_{u}^{2}} \frac{\partial \psi}{\partial z}\right)=\mathrm{PV}_{v} .
$$

This elliptic PDE has a particularly concise form because it does not depend on any of the moist $M$ variables.

To see that inversion with $\mathrm{PV}_{v}$ does not recover the balanced streamfunction, we compare the solution $\psi_{v}$ from $\mathrm{PV}_{v}$ inversion (27) and the solution $\psi_{e}$ from $\mathrm{PV}$-and- $M$ inversion using $\mathrm{PV}_{e}$ in (22). To compare, we take the difference between the two corresponding PDEs to obtain

$$
\mathscr{L}\left(\psi_{e}-\psi_{v}\right)=\frac{1}{f \tilde{\rho}} \frac{\partial}{\partial z}\left[\tilde{\rho}\left(\frac{f^{2}}{N_{s}^{2}}-\frac{f^{2}}{N_{u}^{2}}\right) \frac{g}{\tilde{\theta}}\left(\theta-\theta^{B}\right) H_{s}\right],
$$

where $\theta^{B}$ is the balanced component of the potential temperature arising from the streamfunction as $\theta^{B}=(f \tilde{\theta} / g) \partial \psi_{e} / \partial z$ and the linear operator $\mathscr{B}$ is defined as

$$
\mathscr{B}=\nabla_{H}^{2}+\frac{1}{\tilde{\rho}} \frac{\partial}{\partial z} \tilde{\rho} \frac{f^{2}}{N_{u}^{2}} \frac{\partial}{\partial z} ;
$$

see appendix D for details on the derivation of (28). Since the PDE in (28) is nonhomogeneous (i.e., the right-hand side is nonzero), the streamfunction $\psi_{e}$ obtained from (22) will be different from the solution $\psi_{v}$ of (27), even if the two inversions use identical boundary conditions. Since the right-hand side may become nearly zero in the upper troposphere where the buoyancy frequencies $N_{u}^{2}$ and $N_{s}^{2}$ are nearly equal, one would expect the most pronounced differences to be seen in the lower and middle troposphere. Also note that the key differences arising in the right-hand side are due to unbalanced potential temperature, $\theta-\theta^{B}$, in saturated regions, where $H_{s}=1$. In other words, phase changes of water are the source of the discrepancy between the $\mathrm{PV}_{v}$-derived streamfunction $\psi_{v}$ and the balanced streamfunction $\psi_{e}$.

Why does inversion with $\mathrm{PV}_{v}$ not recover the balanced streamfunction? It is because, for a system with phase changes, $\mathrm{PV}_{v}$ itself is not balanced. To see this, consider the evolution equation for $\mathrm{PV}_{v}$. We may obtain this evolution equation by formally differentiating (26) by the horizontal material derivative $D_{H} / D t$, and using the fact that the buoyancy $b$ is given by (8a) and the evolution equations for $b_{u}$ and $b_{s}$ are (15a) and (15b), respectively. The result is

$$
\begin{aligned}
\frac{D_{H} \mathrm{PV}_{v}}{D t}= & \frac{f}{\tilde{\rho}} \frac{\partial}{\partial z}\left[\frac{\tilde{\rho}}{N_{u}^{2}}\left(N_{u}^{2}-N_{s}^{2}\right) w H_{s}\right. \\
& \left.+\frac{\tilde{\rho}}{N_{u}^{2}} \frac{g \tilde{\gamma}}{\tilde{\theta}}\left(A_{r}+C_{r}-E_{r}\right) H_{u}\right] .
\end{aligned}
$$

Notice the term on the right-hand side that involves $w H_{s}$; it is active in saturated regions, and it arises from cloud latent heating. In broad terms, because of this $w$ term on the right-hand side, $\mathrm{PV}_{v}$ is coupled with waves. Indeed, from a more thorough calculation using a suitable nondimensionalization and distinguished asymptotic limit, one can see that in an unbalanced setting this $w$ term is $O\left(\mathrm{Ro}^{-1}\right)$ for small Rossby numbers, which corresponds to fast wave oscillations, so $\mathrm{PV}_{v}$ is not balanced.

The physical origin of the $w H_{s}$ term in (29) can be seen more clearly from the evolution equation:

$$
\frac{D}{D t}\left(\frac{1}{\tilde{\rho}} \zeta_{a} \cdot \nabla b\right)=\frac{1}{\tilde{\rho}} \zeta_{a} \cdot \nabla \dot{b}
$$

which describes the evolution of a $\mathrm{PV}_{v}$ variable without any assumption of small Froude or small Rossby number. This evolution equation can be derived from the curl of momentum in (1a), using the boldface notation $\boldsymbol{\zeta}_{a}=\nabla \times \mathbf{u}+f \hat{\mathbf{z}}$ for the absolute vorticity vector, and following a derivation similar to that in Schubert et al. (2001). The notation $\dot{b}=D b / d t$ indicates the sources of buoyancy, including those related to phase changes and latent heating. In a setting of small Rossby number, (30) should reduce to (29), and cloud latent heating gives rise to the $w H_{s}$ term in (29).

As an illustration of the unbalanced evolution of $\mathrm{PV}_{v}$, we return the simulations described in section 2 . Figures $3 \mathrm{a}$ and $3 \mathrm{~b}$ show $\mathrm{PV}_{e}(x, y, z, t)$ and $\mathrm{PV}_{v}(x, y, z, t)$, respectively, for times $t=1.0,1.1$, and 1.2; recall that a 0.1 time increment is associated with the fast time scale. The data are shown along the vertical line with constant $x=y=\pi$, so the $\mathrm{PV}_{v}$ is shown along a line through the 3D domain. Over part of the domain, the three curves are nearly overlapping, indicating balanced evolution (i.e., limited evolution over the fast time scale). In the portion of the domain that may be saturated (roughly for heights $1 \leq z \leq 3$ ), however, the $\mathrm{PV}_{v}$ values change substantially from one time to another, indicating unbalanced evolution at these heights. Such behavior is consistent with the $\mathrm{PV}_{v}$ evolution equation shown in (29), which also indicates that $\mathrm{PV}_{v}$ will be influenced by fast waves (the $w$ factor) in regions that are saturated (where the $H_{s}$ factor is nonzero). Similar plots for $\mathrm{PV}_{e}$ in Fig. 3a corroborate the $\mathrm{PV}_{e}$ evolution equation in (19): $\mathrm{PV}_{e}$ is not influenced by waves, and it therefore has an evolution that is balanced (i.e., evolving on the slow time scale). As a statistical measure of the variability, the standard deviation of the $\mathrm{PV}_{e}$ and $\mathrm{PV}_{v}$ fluctuations are shown in Fig. 3c. The $\mathrm{PV}_{v}$ variable has an enhanced standard deviation compared with $\mathrm{PV}_{e}$, an indication of the unbalanced evolution of $\mathrm{PV}_{v}$ in saturated regions. 
(a)

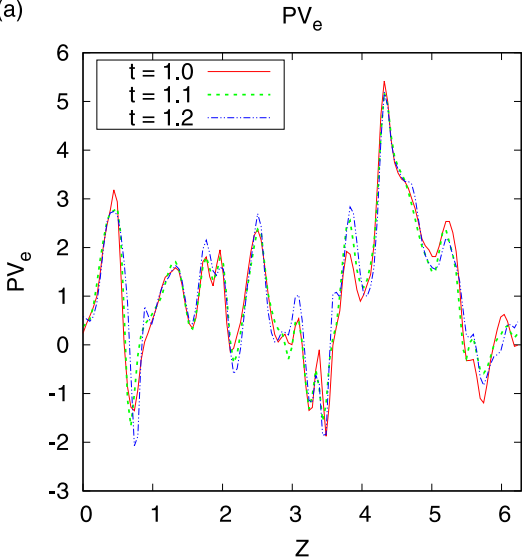

(b)

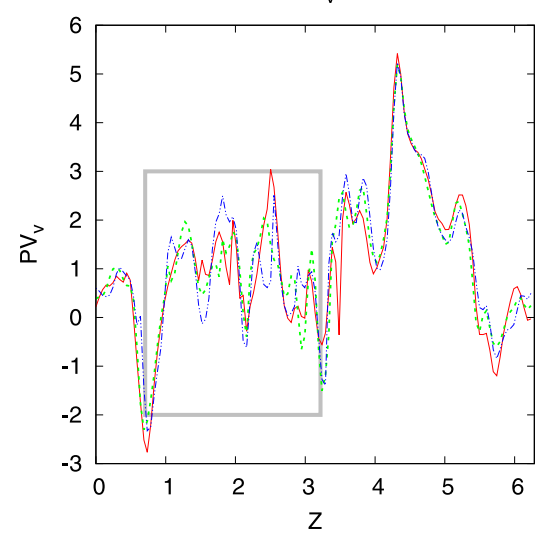

(c)

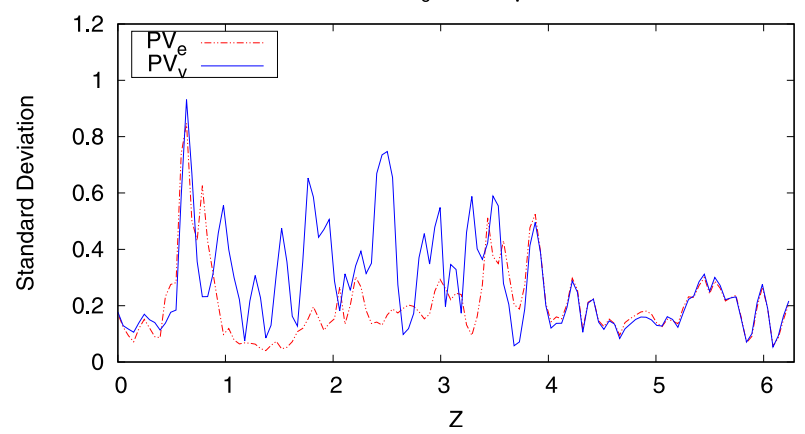

FIG. 3. Illustration of the unbalanced evolution of $\mathrm{PV}_{v}$, the PV variable that is based on virtual potential temperature $\theta_{v}$, and the balanced evolution of $\mathrm{PV}_{e}$, the $\mathrm{PV}$ variable that is based on equivalent potential temperature $\theta_{e}$ : (a) Three snapshots of $\mathrm{PV}_{e}(\pi$, $\pi, z, t$ ), which has been evaluated at $x=y=\pi$ and is shown for three times $t=1.0,1.1$, and 1.2. (b) As in (a), but for $\mathrm{PV}_{v}$. The gray rectangle indicates the region of the moisture bubble and hence the locations that are most likely to be saturated $\left(H_{s}=1\right)$. (c) Standard deviation of $\mathrm{PV}_{e}$ (dashed) and $\mathrm{PV}_{v}$ (solid), where the standard deviation is defined at each spatial location based on the time series of 80 data points between times $t=1.0$ and 1.2.
In summary, PV based on $\theta_{v}$ can be used to recover $a$ streamfunction, but it is not the balanced streamfunction, for a system with phase changes. This is because $\theta_{v}$ and $\theta$ are not conserved variables, since they are influenced by latent heating, and the corresponding PV variables are therefore not balanced.

\section{4. $M$ variables are PV-like: Conserved tracers}

To illustrate two of the ways that the $M$ variables are similar to PV, we use numerical simulations. First, we illustrate that the $M$ variables evolve on a slow time scale. To do so, we return to the idealized simulations of section 2, and we plot the evolution of $M$ at times $t=0$, 0.1 , and 0.2 ; see Fig. 4 . The variable $M$ shows essentially no changes over this fast time scale, since it is a balanced or slowly evolving variable, like PV.

Second, to illustrate the fact that $M$ variables are approximately conserved, we roughly track a parcel in a simplified simulation of midlatitude flow. The simulation is done using the WRF Model (Skamarock et al. 2008), version 3.7.1. The setup of the simulation is that used in Wetzel et al. (2019), so we will only briefly describe it here. The simulation consists of a hemispheric sized channel on a $\beta$ plane. The dimensions of the channel are $12000 \mathrm{~km}$ in the east-west direction, $8000 \mathrm{~km}$ in the south-north direction, and $16 \mathrm{~km}$ in the down-up direction with a horizontal resolution of $25 \mathrm{~km}$ and a vertical resolution of approximately $250 \mathrm{~m}$. For boundary conditions, we choose periodicity in the $x$ (east-west) direction and specified, or rigid, in the south and north boundaries such that a temperature and moisture gradient exists from south to north. The Kessler (1969) microphysics scheme is used, which contains warm moisture constituents of rainwater, cloud water, and water vapor. We use no shortwave or longwave radiation, no surface or boundary layer physics, and no cumulus parameterization schemes.

In Fig. 5, we show snapshots of the quantities $M_{r}, \mathrm{PV}_{e}$, and moisture $q_{t}$ over a timespan of 1 day in the channel simulation. In particular, we show day 91 and day 92 after the start of the simulation, where equilibration of the turbulent flow is achieved at roughly 30 days after the start of the simulation. We immediately note that the $\mathrm{PV}$ and $M$ variables $\mathrm{PV}_{e}$ and $M_{r}$ share broad bulk features. Namely, both variables contain roughly uniform regions, where $\mathrm{PV}_{e}$ takes a value of roughly $2-4 \mathrm{~s}^{-1}$ uniformly over a large northern region and a value from -2 to $-4 \mathrm{~s}^{-1}$ uniformly over a large southern region; also, $M_{r}$ takes a value from roughly -25 to $-35 \mathrm{~g} \mathrm{~kg}^{-1}$ uniformly over a large northern region and from 35 to $45 \mathrm{~g} \mathrm{~kg}^{-1}$ uniformly over a large southern region. The two uniform regions are separated by a transition zone or sharp 

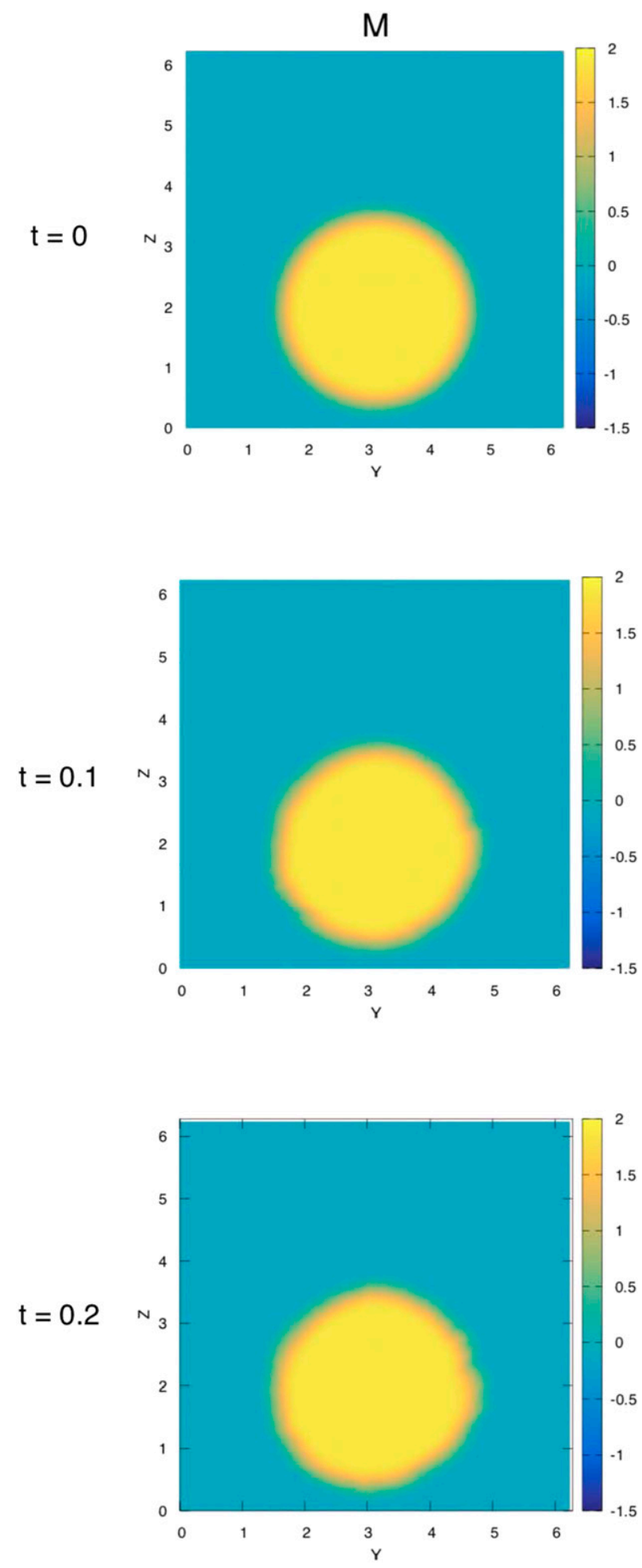

FIG. 4. Similar to Fig. 1, but for the balanced and slowly evolving variable $M$. gradient aligned with the zonal jet in the balanced flow. Moreover, each variable appears to mostly advect its features about the flow; note, in particular, the $M_{r}$ eddies that are advected on the north side of the jet, for example, at $(x, y) \approx(4500 \mathrm{~km}, 7000 \mathrm{~km})$ on day 91 .

The $q_{t}$ variable, on the other hand, while it shares in the presence of a transition region, contains large concentrations of moisture that do not appear to be simply advected by the flow, but are rather combined and disseminated. To test this fact more carefully, we approximately track the variables $M_{r}, \mathrm{PV}_{e}$, and $q_{t}$ on a parcel denoted by a red circle in Fig. 5. The parcel is taken from a starting location at 91 days and then allowed to freely advect using the balanced flow at days 91 and 91.5 until day 92. At each snapshot, we average the variable values over a square box of dimensions $50 \mathrm{~km} \times 50 \mathrm{~km}$ centered at the parcel location shown. The results of following this parcel, which have been normalized by the largest value that the box takes over the timespan, are shown in Fig. 6. We note that over this one day, the conserved variables of $\mathrm{PV}_{e}, M_{r}$ change about $15 \%$ from their maximum value, while the $q_{t}$ variable undergoes a drop off of over $40 \%$ as we follow this parcel. This indicates that the variables $\mathrm{PV}_{e}$ and $M_{r}$ remain approximately constant over the evolution of the parcel than the variable $q_{t}$, even in a region with significant moisture. This reaffirms our understanding that the PV and $M$ variables act as conserved quantities of the flow (at least approximately, given the influence of microphysical source terms, etc.).

\section{Distinguishing characteristics of $M$ variables}

The $M$ variables have a number of defining characteristics that differentiate them from other thermodynamic variables.

First, by construction, the $M$ variables have no background states. That is, they are merely defined as arising from anomalies—see, for example, (13b) and (13c) — and therefore have no obvious reference state. Indeed, the fact that the $M$ variables do not have a background state can be immediately surmised from the lack of a $w$ term, multiplied by the gradient of the respective background state, in their evolution equations; see, for example, (14b) and $(14 \mathrm{c})$ and compare these with the evolution equations for other thermodynamics variables (12b) and (12c).

Second, because the lack of $w$ terms in their evolution equations, the $M$ variables are not coupled to (inertiogravity) waves. Therefore, the $M$ variables are balanced variables.

Third, the $M$ variables may resemble the variables $q_{t}, q_{t}-q_{r}$, or $\theta_{e}$ at certain altitudes depending on the relative weakness of the background state gradients 

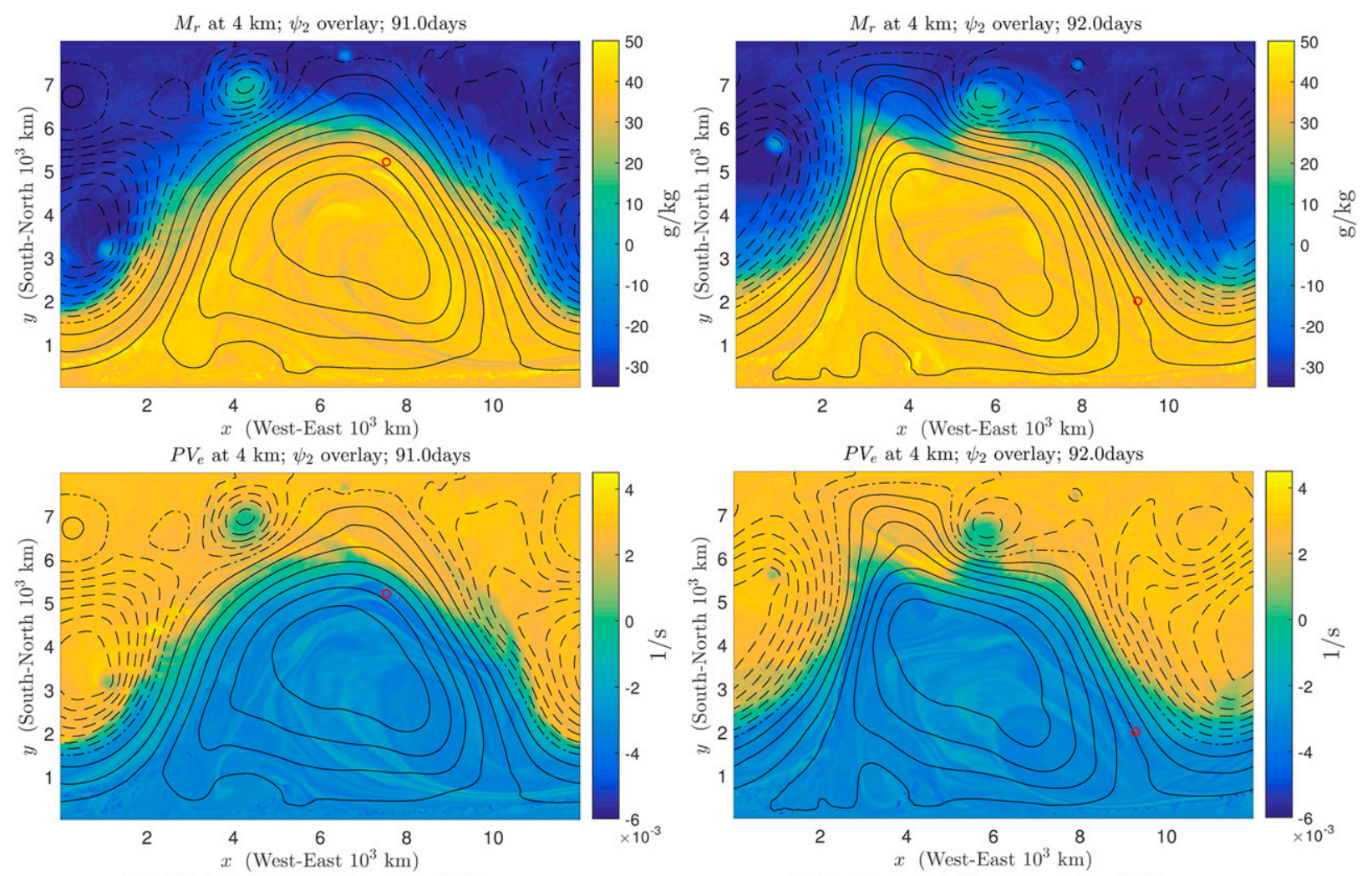

Total Water $q_{t}$ at $4 \mathrm{~km} ; \psi_{2}$ overlay; 91.0days
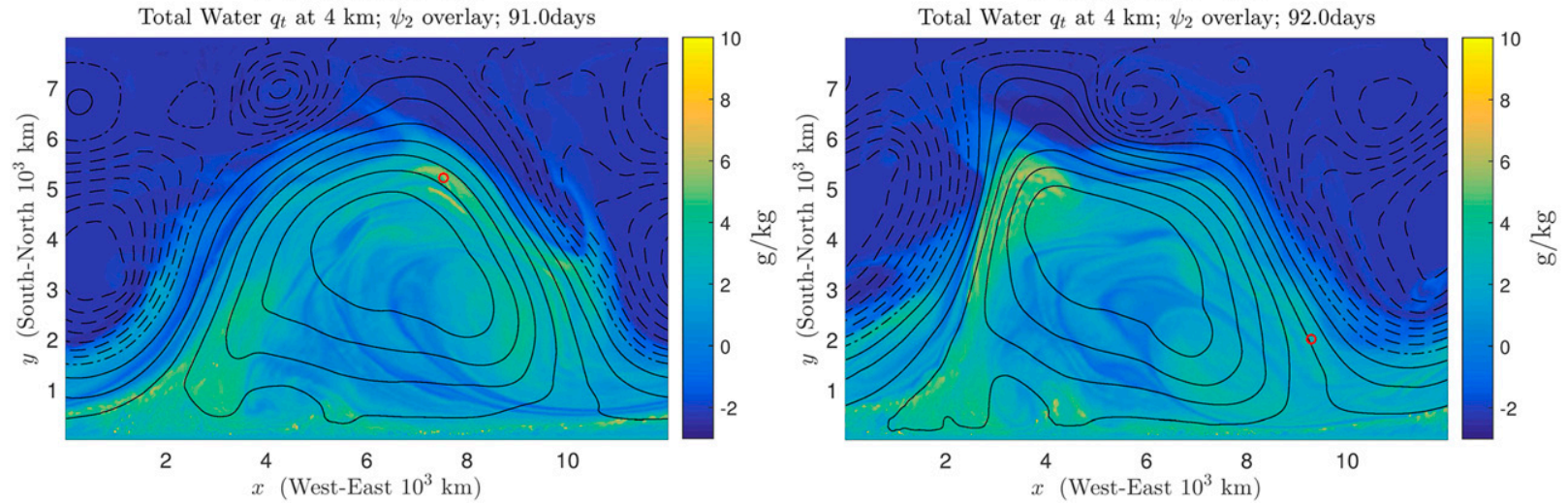

FIG. 5. Snapshots of $M$ variable $M_{r}, \mathrm{PV}$ variable $\mathrm{PV}_{e}$, and moisture variable $q_{t}$ with balanced streamfunction overlay at 4-km height between 91 and 92 days; solid lines denote positive streamfunction; dashed lines denote negative streamfunction. The advected parcel is represented by the red circle.

associated with the thermodynamic variables. For example, the $M$ variable $M_{r}$, defined in (13c), weights the two variables $q_{t}-q_{r}$ and $\theta_{e}$ using the background gradient ratio $\tilde{G}_{M}=-\left(d \tilde{q}_{t} / d z\right) /\left(d \tilde{\theta}_{e} / d z\right)$. In the atmosphere, we expect the moisture variable $q_{t}$ to have a small background gradient state $d \tilde{q}_{t} / d z$ at high altitudes and a large background gradient at low altitudes because of the large concentration of moisture near the surface and scarcity of moisture from mid- to high altitudes. Similarly, the equivalent potential temperature $\theta_{e}$ is expected to have a smaller background gradient state $d \tilde{\theta}_{e} / d z$ at lower altitudes in midlatitudes. Therefore, $M_{r} \approx \tilde{G}_{M} \theta_{e}$ at low altitudes and $M_{r} \approx q_{t}-q_{r}$ at higher altitudes for a common atmospheric setup. Indeed, we observe just such a situation in our midlatitude channel simulation; see Fig. 7. Note that Fig. 7 shows that $M_{r}$ resembles $\tilde{G}_{M} \theta_{e}$ at the $2-\mathrm{km}$ height, where $\tilde{G}_{M} \approx$ $1.1 \mathrm{~g} \mathrm{~kg}^{-1} \mathrm{~K}^{-1}$ at this height, while $M_{r}$ resembles $q_{t}-q_{r}$ at the 8 - $\mathrm{km}$ height, with $\tilde{G}_{M} \approx 5 \times 10^{-3} \mathrm{~g} \mathrm{~kg}^{-1} \mathrm{~K}^{-1}$ at this height.

Fourth, the $M$ variables are associated with an additional component of the total energy (Marsico et al. 2019). 


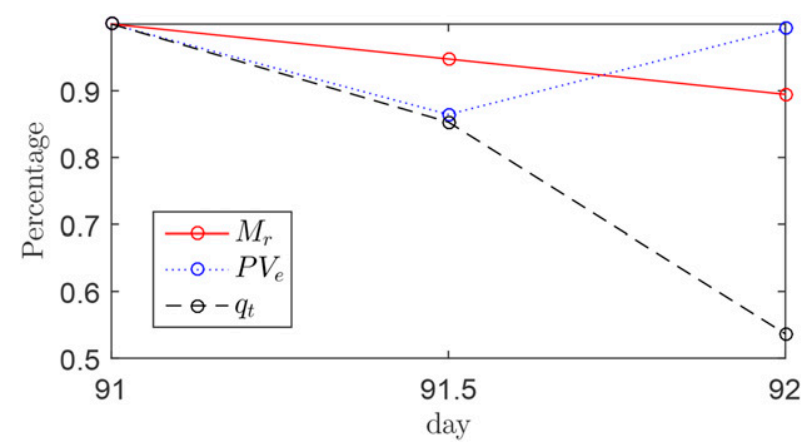

FIG. 6. Percentage change of variables $M_{r}, \mathrm{PV}_{e}$, and $q_{t}$ while following a parcel advected by the balanced flow. The location of the parcel is shown by a red dot in Fig. 5 .

Beyond the buoyant potential energy, a moist latent energy is also present, and it could be written in the form $H_{u} M^{2}$. In the Boussinesq case, it corresponds to our presentation of $M=q_{t}+\tilde{G}_{M} \theta_{e}$. In the anelastic case, on the other hand, the energetics suggest a definition of an $M$ variable as

$$
\begin{aligned}
M_{\text {energy }}= & \left\{\int_{z_{\mathrm{lnb}, u}}^{z_{\mathrm{lcl}}}\left[b_{u}^{\mathrm{tot}}-\tilde{b}_{u}\left(z^{\prime}\right)\right] d z^{\prime}\right. \\
& \left.-\int_{z_{\mathrm{ln} b, s}}^{z_{\mathrm{lcl}}}\left[b_{s}^{\mathrm{tot}}-\tilde{b}_{s}\left(z^{\prime}\right)\right] d z^{\prime}\right\}^{1 / 2},
\end{aligned}
$$

where the integral is a type of "partial integration" where $b_{u}^{\text {tot }}$ and $b_{s}^{\text {tot }}$ are held fixed [ $M_{\text {energy }}$ was called $M_{\text {anelastic }}$ by Marsico et al. (2019)]. Here the background states are defined as

$$
\begin{aligned}
& \tilde{b}_{u}(z)=\int_{0}^{z} N_{u}^{2}\left(z^{\prime}\right) d z^{\prime} \text { and } \\
& \tilde{b}_{s}(z)=\int_{0}^{z} N_{s}^{2}\left(z^{\prime}\right) d z^{\prime},
\end{aligned}
$$

and the "total" variables are defined as

$$
\begin{aligned}
& b_{u}^{\mathrm{tot}}=\tilde{b}_{u}(z)+b_{u} \quad \text { and } \\
& b_{s}^{\mathrm{tot}}=\tilde{b}_{s}(z)+b_{s} .
\end{aligned}
$$

The bounds of integration in (31) include $z_{\operatorname{lnb}, u}$ and $z_{\text {lnb }, s}$, which correspond to levels of neutral buoyancy (lnb), with respect to $N_{u}$ and $N_{s}$, respectively, and are defined as the solutions to

$$
\tilde{b}_{u}\left(z_{\operatorname{lnb}, u}\right)=b_{u}^{\text {tot }} \quad \text { and } \quad \tilde{b}_{s}\left(z_{\operatorname{lnb}, u}\right)=b_{s}^{\text {tot }},
$$

with $b_{u}^{\text {tot }}$ and $b_{s}^{\text {tot }}$ taken to be fixed values. The other bound of integration, $z_{\mathrm{lc}}$, is similar to a lifted condensation level (lcl) as it is defined as the solution of

$$
b_{u}^{\mathrm{tot}}-b_{s}^{\mathrm{tot}}=\tilde{b}_{u}\left(z_{\mathrm{lcl}}\right)-\tilde{b}_{s}\left(z_{\mathrm{lcl}}\right),
$$

with $b_{u}^{\text {tot }}$ and $b_{s}^{\text {tot }}$ again taken to be fixed values.

Our final comments on $M$ variables will be with regard to the energetically motivated definition of $M_{\text {energy }}$ in (31). The $M_{\text {energy }}$ variable in (31) is a material invariant, not only in the limit of small Froude and Rossby numbers like $M_{r}$, but also in general for any Froude and Rossby numbers. Hence, $M_{\text {energy }}$ is like Ertel PV. It obeys

$$
\frac{D}{D t} M_{\text {energy }}=0
$$

where the full material derivative $D / D t$ is used, in contrast to the horizontal material derivative that comes in the small Froude and Rossby case for $M_{r}$ advection in (14c). ${ }^{4}$ To see this material invariant property of $M_{\text {energy, }}$, note from (31)-(35) that $M_{\text {energy }}$ is a function of $b_{u}^{\text {tot }}$ and $b_{s}^{\text {tot }}$ alone (since $z_{\mathrm{lcl}}, z_{\operatorname{lnb}, u}$, and $z_{\operatorname{lnb}_{s}}$ are themselves also functions of $b_{u}^{\text {tot }}$ and $b_{s}^{\text {tot }}$ alone), and $b_{u}^{\text {tot }}$ and $b_{s}^{\text {tot }}$ are themselves material invariants, from (15), or from the more complete description (not shown) based on (8), in the case that warm-rain microphysical source terms are neglected.

Last, we consider a possible answer to the question: What is $M$ ? What is a physically intuitive viewpoint of $M$ (beyond earlier descriptions of $M$ as, e.g., the thermodynamic quantity that is a material invariant and that has zero vertical background gradient)? The energybased $M_{\text {energy }}$ in (31) offers some possible intuition: $M_{\text {energy }}$ is like convective available potential energy (CAPE; Moncrieff and Miller 1976; Emanuel 1994; Hernández-Dueñas et al. 2019). In particular, it is defined as a vertical integral of buoyancy, from a parcel's lifted condensation level to its level of neutral buoyancy (albeit with some added complexity here with two buoyancies, $b_{u}^{\text {tot }}$ and $b_{s}^{\text {tot }}$, two lcls, etc.). In this paper, we instead used $M_{r}$ as a typical $M$ variable because it offers a simpler definition mathematically as a linear combination of $q_{t}$ and $\theta_{e}$, and simpler formulas and derivations of PV-and- $M$ inversion, and so on. Nevertheless, it would be interesting in the future to explore the quantity $M_{\text {energy }}$ for its potentially valuable physical interpretation as a CAPE-like quantity.

\section{Discussion and conclusions}

In this paper, we investigated the decomposition of midlatitude moist flows into balanced and unbalanced

\footnotetext{
${ }^{4}$ Note that these statements about material invariants are neglecting warm-rain microphysical source terms although not neglecting phase changes between water vapor and cloud water.
} 

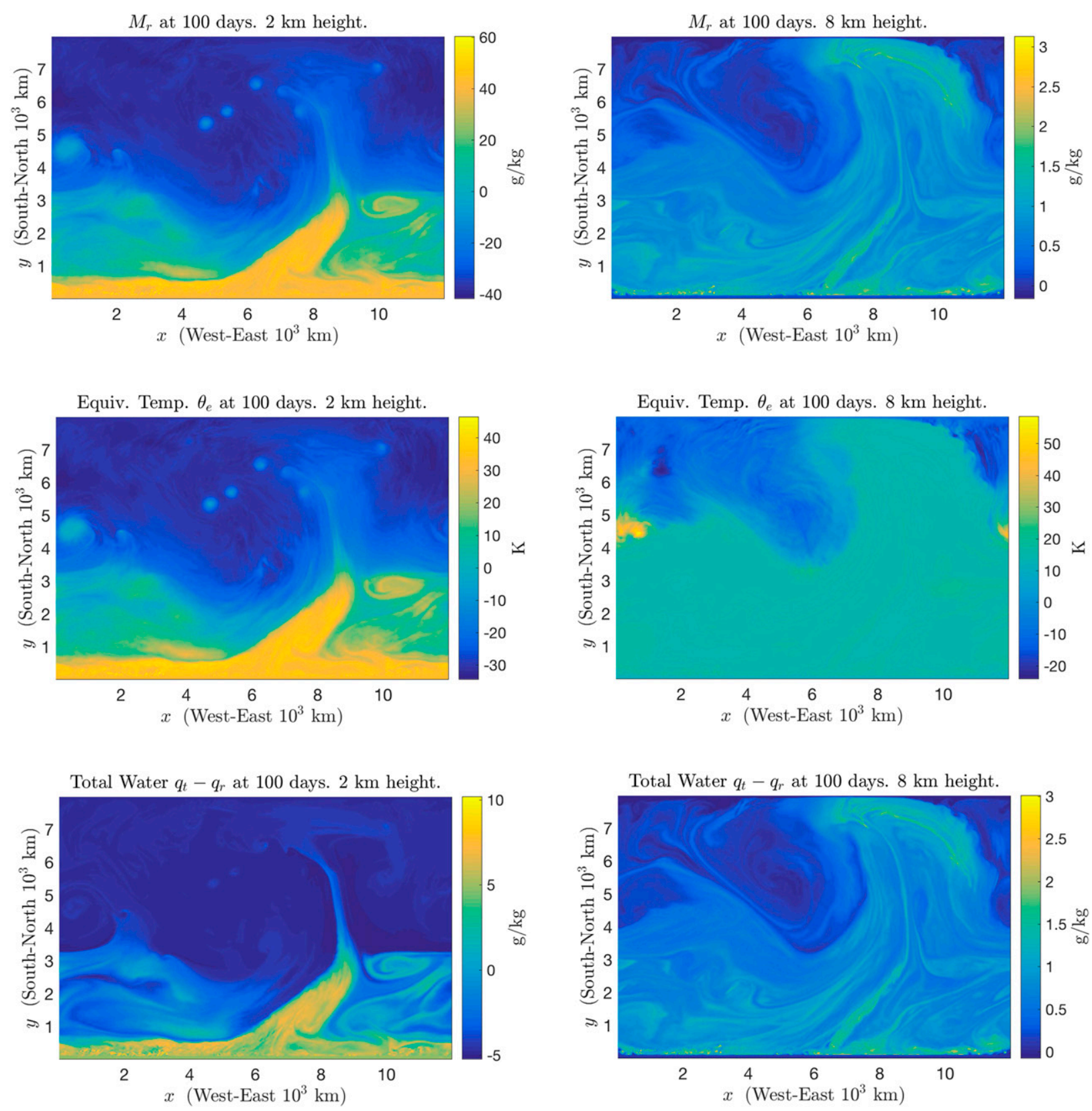

FIG. 7. Snapshots of $M_{r}, \theta_{e}$, and $q_{t}-q_{r}$ at 2- and 8-km height on 100 days.

components. This decomposition was accomplished using a recently introduced inversion principle, called $\mathrm{PV}$-and- $M$ inversion, to diagnostically recover the moist balanced flow of the system (Smith and Stechmann 2017; Wetzel et al. 2019). PV-and- $M$ inversion is a moist generalization of dry-air inversion principles. In an absolutely dry atmosphere, only a single variable, $\mathrm{PV}$, is sufficient to recover the balanced flow. In moist flows, however, additional balanced modes not present in absolutely dry dynamics become dynamically significant and need to be retained to successfully describe the evolution of the balanced flow. Namely, the addition of moisture leads to significant additional balanced modes. The balanced flow of a moist system is then no longer one-dimensional but multidimensional (i.e., it contains both PV and $M$ modes).

Several subtle points of moist PV inversion have been pointed out in previous studies, and here we discussed some of these points from the perspective of $\mathrm{PV}$-and- $M$ inversion. For instance, it has been pointed out that 
traditional PV inversion cannot be carried out using the potential vorticity $\mathrm{PV}_{e}$ that is based on $\theta_{e}$ (unless saturated conditions are assumed; see, e.g., Cao and Cho 1995; Schubert et al. 2001). Here, we described how this issue can be remedied by the inclusion of the moist balanced modes to the inversion principle (i.e., by using $\mathrm{PV}$-and- $M$ inversion). Namely, inversion principles using $\mathrm{PV}_{e}$ may be constructed once the moist $M$ modes are included, and PV-and- $M$ inversion may then be used to recover all relevant balanced variables. Indeed, we find that $\mathrm{PV}$-and- $M$ inversion may be equivalently carried out using different families of PV variables. As a second subtle point, we showed that it is possible for a PV variable to have a traditional PV inversion principle, even though the PV variable is not balanced; in this case, the PV inversion can be carried out, but it does not recover the balanced flow. For example, due to phase changes, the $\mathrm{PV}_{v}$ variable-derived using the virtual potential temperature-is coupled with waves and therefore is not balanced. This makes an inversion principle using $\mathrm{PV}_{v}$ unsuitable to recover the balanced component of the flow.

Another purpose of this paper was to explore the properties of the $M$ modes. The $M$ modes themselves qualitatively behave as traditional PV variables in that they are material invariants or, equivalently, they are tracers advected by the flow. As they are uncoupled from waves, the $M$ modes have a zero vertical background gradient. Indeed, we find that the $M$ variables closely track thermodynamic variables at different altitudes depending on the background gradient. For example, in the case of $M_{r}$, we find that $M_{r} \approx \tilde{G}_{M} \theta_{e}$ at a $2 \mathrm{~km}$ height, while $M_{r} \approx q_{t}-q_{r}$ at $8 \mathrm{~km}$ where the background gradient of moisture is negligible. Namely, the $M$ mode $M_{r}$ closely resembles the equivalent temperature at low altitudes, where $\tilde{G}_{M} \theta_{e}$ is approximately a conserved variable, and resembles the total moisture at higher altitudes, where the moisture is approximately a conserved variable. ${ }^{5}$ Last, a conceptually useful physical interpretation of the $M$ modes is that they are related to convective available potential energy; an additional component of total energy arising from the presence of moisture. A deeper exploration of the connection between $M$ modes with energy is, however, left to a future paper.

Acknowledgments. The authors thank Rupert Klein and an anonymous reviewer for helpful comments.

\footnotetext{
${ }^{5}$ In all cases we use warm-rain (Kessler) microphysics, as a simple version of microphysics, but other more comprehensive microphysics could also be used so as to account for more realistic hydrometeors.
}

Partial support for this research was provided by National Science Foundation Grants AGS-1443325 and DMS-1907667, and by the University of WisconsinMadison Office of the Vice Chancellor for Research and Graduate Education with funding from the Wisconsin Alumni Research Foundation. This research was performed using the computer resources and assistance of the UW-Madison Center For High Throughput Computing (CHTC) in the Department of Computer Sciences. The CHTC is supported by UW-Madison, the Advanced Computing Initiative, the Wisconsin Alumni Research Foundation, the Wisconsin Institutes for Discovery, and the National Science Foundation and is an active member of the Open Science Grid, which is supported by the National Science Foundation and the U.S. Department of Energy's Office of Science.

\section{APPENDIX A}

\section{Boussinesq Equations}

The system of equations used in the numerical simulation discussed in section 2 are as follows. The Boussinesq equations with Coriolis terms for a moist atmosphere with two moisture constituents are

$$
\begin{aligned}
\frac{D \mathbf{u}}{D t}+f \hat{\mathbf{z}} \times \mathbf{u} & =-\nabla\left(\frac{p}{\rho_{0}}\right)+\hat{\mathbf{z}} b, \\
\nabla \cdot \mathbf{u} & =0, \\
\frac{D \theta_{e}}{D t}+w \frac{d \tilde{\theta}_{e}}{d z} & =0, \quad \text { and } \\
\frac{D q_{t}}{D t}+w \frac{d \tilde{q}_{t}}{d z} & =0
\end{aligned}
$$

where $\rho_{0}$ is a constant reference density. All other variables names are the same as those used in the anelastic system of section 3. The Boussinesq equations constitute a special case of the anelastic equations of section 3 under the assumption of constant reference density. It is also assumed here that water is in the form of two types-water vapor and cloud water-without rainwater and associated microphysical processes. Such a nonprecipitating setup is a simple case that still includes moisture and phase changes.

\section{APPENDIX B}

\section{Balanced Component of Moisture}

The balanced component of total water $q_{t}$ is directly determined by the balanced variables $\psi, \mathrm{PV}_{e}, M$, and/or 
$M_{r}$. A formula for the balanced moisture $q_{t}^{B}$ can be found as follows; the superscript $B$ will denote balanced components. Equation (13b) can be understood in terms of only balanced components to solve for $q_{t}^{B}$. Namely, using the balanced $M$ and $\theta_{e}^{B}$ variables, the balanced $q_{t}$ is given by

$$
q_{t}^{B}=M-\tilde{G}_{M} \theta_{e}^{B} .
$$

Therefore, it remains to deduce how the balanced $\theta_{e}$ depends on the balanced PV $-M$ variables. To do this, we may readily use (21b). That is,

$$
\theta_{e}^{B}=\frac{N_{s}^{2}}{N_{u}^{2}}\left(\theta^{B}+\tilde{\gamma} M_{r}\right) H_{u}+\left(\theta^{B}+\tilde{\gamma} q_{v s}\right) H_{s}
$$

in terms of the balanced temperature $\theta^{B}$ and balanced $M_{r}$. We note that the balanced temperature $\theta^{B}$ may be determined using the streamfunction using (10) and (18b) to obtain $g \theta^{B} / \tilde{\theta}=f \partial \psi / \partial z$. Then, the balanced moisture is

$$
q_{t}^{B}=M-\tilde{G}_{M}\left[\frac{N_{s}^{2}}{N_{u}^{2}}\left(\theta^{B}+\tilde{\gamma} M_{r}\right) H_{u}+\left(\theta^{B}+\tilde{\gamma} q_{v s}\right) H_{s}\right],
$$

in terms of balanced $\theta, M$, and $M_{r}$. All other variables in the equation, except for the known indicator functions $H_{u}$ and $H_{s}$, depend only on the vertical height $z$ and are therefore prescribed from the background state of the system.

\section{APPENDIX C}

\section{Difference between $P V_{e}$ and $P V_{u}$ Inversions}

The difference between the inversion principle (22) for $\mathrm{PV}_{e}$ and (24) for $\mathrm{PV}_{u}$, assuming that the unsaturated and saturated regions are the same from each inversion, gives

$$
\begin{aligned}
\mathscr{A}\left(\psi_{e}-\psi_{u}\right)= & \mathrm{PV}_{e}-\mathrm{PV}_{u}-\frac{1}{\tilde{\rho}} \frac{\partial}{\partial z}\left(\tilde{\rho} \frac{f^{2}}{N_{u}^{2}} \frac{g \tilde{\gamma}}{f \tilde{\theta}} M_{r} H_{u}\right. \\
& \left.+\tilde{\rho} \frac{f^{2}}{N_{s}^{2}} \frac{g \tilde{\gamma}}{f \tilde{\theta}} q_{u s} H_{s}+\tilde{\rho} f M_{b} H_{s}\right)
\end{aligned}
$$

where the operator $\mathscr{A}$ is defined by (25b). The righthand side, however, may seen to be identically zero. From definition (13a) for $\mathrm{PV}_{e}$ and (16a) for $\mathrm{PV}_{u}$ we find

$$
\mathrm{PV}_{e}-\mathrm{PV}_{u}=\frac{f}{\tilde{\rho}} \frac{\partial}{\partial z}\left(\frac{\tilde{\rho}}{d \tilde{\theta}_{e} / d z} \theta_{e}\right)-\frac{f}{\tilde{\rho}} \frac{\partial}{\partial z}\left(\frac{\tilde{\rho}}{N_{u}^{2}} b_{u}\right) .
$$

Now, note that (16b) and (10) may be used in each phase to deduce the formula

$$
\frac{b_{u}}{N_{u}^{2}}=\frac{1}{N_{u}^{2}} g \frac{\theta}{\tilde{\tilde{\theta}}} H_{u}-\left(M_{b}-\frac{1}{N_{s}^{2}} g \frac{\theta}{\tilde{\theta}}\right) H_{s} .
$$

Combining this with (21b) makes the right-hand side of (C2) become

$$
\begin{aligned}
& \frac{1}{d \tilde{\theta}_{e} / d z} \theta_{e}-\frac{1}{N_{u}^{2}} b_{u} \\
& =\frac{1}{d \tilde{\theta} / d z}\left(\theta+\tilde{\gamma} M_{r}\right) H_{u}+\frac{1}{d \tilde{\theta} / d z}\left(\theta+\tilde{\gamma} q_{v s}\right) H_{s} \\
& \quad-\frac{1}{N_{u}^{2}} g \frac{\theta}{\tilde{\theta}} H_{u}+\left(M_{b}-\frac{1}{N_{s}^{2}} g \frac{\theta}{\tilde{\theta}}\right) H_{s} .
\end{aligned}
$$

Using the definition of the background frequency (15c) and the fact that the same $\theta$ is used in each inversion, we are able to simply show that the right-hand side of $(\mathrm{C} 1)$ is identically zero.

\section{APPENDIX D}

\section{Difference between $\mathbf{P V}_{e}$ and $\mathbf{P V}$ v Inversions}

The difference between the inversion principle (22) for $\mathrm{PV}_{e}$ and (27) for $\mathrm{PV}_{v}$ gives

$$
\begin{aligned}
& \mathscr{L}\left(\psi_{e}-\psi_{v}\right)+\frac{1}{\tilde{\rho}} \frac{\partial}{\partial z}\left[\tilde{\rho}\left(\frac{f^{2}}{N_{s}^{2}}-\frac{f^{2}}{N_{u}^{2}}\right) H_{s} \frac{\partial \psi_{e}}{\partial z}\right] \\
& =\mathrm{PV}_{e}-\mathrm{PV}_{v}-\frac{1}{\tilde{\rho}} \frac{\partial}{\partial z}\left[\tilde{\rho} \frac{g \tilde{\gamma}}{f \tilde{\theta}}\left(\frac{f^{2}}{N_{u}^{2}} M_{r} H_{u}+\frac{f^{2}}{N_{s}^{2}} q_{v s} H_{s}\right)\right],
\end{aligned}
$$

where $\mathscr{L}$ is defined in (28b). Now, using the definitions of $\mathrm{PV}_{e}$ in (19) and $\mathrm{PV}_{v}$ in (26), along with (10), we obtain

$$
\begin{aligned}
\mathscr{D}\left(\psi_{e}-\psi_{v}\right)+\frac{1}{\tilde{\rho}} \frac{\partial}{\partial z}\left[\tilde{\rho}\left(\frac{f^{2}}{N_{s}^{2}}-\frac{f^{2}}{N_{u}^{2}}\right) H_{s} \frac{\partial \psi_{e}}{\partial z}\right] \\
=\frac{f}{\tilde{\rho}} \frac{\partial}{\partial z}\left(\frac{g \tilde{\rho}}{N_{s}^{2}} \frac{\theta_{e}}{\tilde{\theta}}\right)-\frac{f}{\tilde{\rho}} \frac{\partial}{\partial z}\left(\frac{g \tilde{\rho}}{N_{u}^{2}} \frac{\tilde{\tilde{\theta}}}{\tilde{\theta}}\right) \\
-\frac{1}{\tilde{\rho}} \frac{\partial}{\partial z}\left[\tilde{\rho} \frac{g \tilde{\gamma}}{f \tilde{\theta}}\left(\frac{f^{2}}{N_{u}^{2}} M_{r} H_{u}+\frac{f^{2}}{N_{s}^{2}} q_{v s} H_{s}\right)\right] .
\end{aligned}
$$

We may then use the definitions (2b) and (13b) to simplify this expression to

$$
\begin{gathered}
\mathscr{L}\left(\psi_{e}-\psi_{v}\right)+\frac{1}{\tilde{\rho}} \frac{\partial}{\partial z}\left[\tilde{\rho}\left(\frac{f^{2}}{N_{s}^{2}}-\frac{f^{2}}{N_{u}^{2}}\right) H_{s} \frac{\partial \psi_{e}}{\partial z}\right] \\
=\frac{f}{\tilde{\rho}} \frac{\partial}{\partial z}\left[\left(\frac{\tilde{\rho}}{N_{s}^{2}}-\frac{\tilde{\rho}}{N_{u}^{2}}\right) \frac{g \theta}{\tilde{\theta}} H_{s}\right] .
\end{gathered}
$$


Last, defining the variable $\partial \psi_{e} / \partial z$ as the balanced temperature, $g\left(\theta^{B} / \tilde{\theta}\right)=f\left(\partial \psi_{e} / \partial z\right)$, gives the desired result (28).

\section{REFERENCES}

Bennetts, D. A., and B. J. Hoskins, 1979: Conditional symmetric instability-A possible explanation for frontal rainbands. Quart. J. Roy. Meteor. Soc., 105, 945-962, https://doi.org/ 10.1002/qj.49710544615.

Brennan, M. J., and G. M. Lackmann, 2005: The influence of incipient latent heat release on the precipitation distribution of the 24-25 January 2000 U.S. East Coast cyclone. Mon. Wea. Rev., 133, 1913-1937, https://doi.org/10.1175/MWR2959.1.

— - — , and K. M. Mahoney, 2008: Potential vorticity (PV) thinking in operations: The utility of nonconservation. Wea. Forecasting, 23, 168-182, https://doi.org/10.1175/2007WAF2006044.1.

Büeler, D., and S. Pfahl, 2017: Potential vorticity diagnostics to quantify effects of latent heating in extratropical cyclones. Part I: Methodology. J. Atmos. Sci., 74, 3567-3590, https:// doi.org/10.1175/JAS-D-17-0041.1.

Cao, Z., and H.-R. Cho, 1995: Generation of moist potential vorticity in extratropical cyclones. J. Atmos. Sci., 52, 3263-3282, https://doi.org/10.1175/1520-0469(1995)052<3263:GOMPVI > 2.0.CO;2.

Chen, S., and S. N. Stechmann, 2016: Nonlinear traveling waves for the skeleton of the Madden-Julian oscillation. Commun. Math. Sci., 14, 571-592, https://doi.org/10.4310/CMS.2016.v14.n2.a11.

Davis, C. A., and K. A. Emanuel, 1991: Potential vorticity diagnostics of cyclogenesis. Mon. Wea. Rev., 119, 1929-1953, https://doi.org/10.1175/1520-0493(1991)119<1929:PVDOC> 2.0.CO;2.

Durran, D. R., and J. B. Klemp, 1982: On the effects of moisture on the Brunt-Väisälä frequency. J. Atmos. Sci., 39, 2152-2158, https://doi.org/10.1175/1520-0469(1982)039<2152:OTEOMO > 2.0.CO;2.

Emanuel, K. A., 1979: Inertial instability and mesoscale convective systems. Part I: Linear theory of inertial instability in rotating viscous fluids. J. Atmos. Sci., 36, 2425-2449, https://doi.org/ 10.1175/1520-0469(1979)036<2425:IIAMCS >2.0.CO;2.

_ 1994: Atmospheric Convection. Oxford University Press, $580 \mathrm{pp}$.

Ertel, H., 1942: Ein neuer hydrodynamischer wirbelsatz. Meteor. Z., 59, 277-281.

Frierson, D. M. W., A. J. Majda, and O. M. Pauluis, 2004: Large scale dynamics of precipitation fronts in the tropical atmosphere: A novel relaxation limit. Commun. Math. Sci., 2, 591-626, https://doi.org/10.4310/CMS.2004.v2.n4.a3.

Gao, S., X. Wang, and Y. Zhou, 2004: Generation of generalized moist potential vorticity in a frictionless and moist adiabatic flow. Geophys. Res. Lett., 31, L12113, https://doi.org/10.1029/ 2003 GL019152.

Grabowski, W. W., and P. K. Smolarkiewicz, 1996: Two-time-level semi-Lagrangian modeling of precipitating clouds. Mon. Wea. Rev., 124, 487-497, https://doi.org/10.1175/1520-0493(1996) $124<0487:$ TTLSLM $>2.0$. CO;2.

Hernández-Dueñas, G., A. J. Majda, L. M. Smith, and S. N. Stechmann, 2013: Minimal models for precipitating turbulent convection. J. Fluid Mech., 717, 576-611, https://doi.org/ 10.1017/jfm.2012.597.

_ - L. M. Smith, and S. N. Stechmann, 2019: Weak- and strongfriction limits of parcel models: Comparisons and stochastic convective initiation time. Quart. J. Roy. Meteor. Soc., 145, 2272-2291, https://doi.org/10.1002/qj.3557.

Hoskins, B. J., M. E. McIntyre, and A. W. Robertson, 1985: On the use and significance of isentropic potential vorticity maps. Quart. J. Roy. Meteor. Soc., 111, 877-946, https://doi.org/ 10.1002/qj.49711147002.

Kessler, E., 1969: On the Distribution and Continuity of Water Substance in Atmospheric Circulations. Meteor. Monogr., No. 32, Amer. Meteor. Soc., 84 pp.

Klein, R., and A. Majda, 2006: Systematic multiscale models for deep convection on mesoscales. Theor. Comput. Fluid Dyn., 20, 525-551, https://doi.org/10.1007/s00162-006-0027-9.

Lackmann, G., 2002: Cold-frontal potential vorticity maxima, the low-level jet, and moisture transport in extratropical cyclones. Mon. Wea. Rev., 130, 59-74, https://doi.org/10.1175/15200493(2002)130<0059:CFPVMT>2.0.CO;2.

—, 2011: Midlatitude Synoptic Meteorology: Dynamics, Analysis, and Forecasting. Amer. Meteor. Soc., 345 pp.

Lipps, F. B., and R. S. Hemler, 1982: A scale analysis of deep moist convection and some related numerical calculations. J. Atmos. Sci., 39, 2192-2210, https://doi.org/10.1175/1520-0469(1982) $039<2192$ :ASAODM $>2.0 . \mathrm{CO} ; 2$.

Madonna, E., H. Wernli, H. Joos, and O. Martius, 2014: Warm conveyor belts in the ERA-Interim dataset (1979-2010). Part I: Climatology and potential vorticity evolution. J. Climate, 27, 3-26, https://doi.org/10.1175/JCLI-D-12-00720.1.

Marquet, P., 2014: On the definition of a moist-air potential vorticity. Quart. J. Roy. Meteor. Soc., 140, 917-929, https:// doi.org/10.1002/qj.2182.

Marsico, D. H., L. M. Smith, and S. N. Stechmann, 2019: Energy decompositions for moist Boussinesq and anelastic equations with phase changes. J. Atmos. Sci., 76, 3569-3587, https:// doi.org/10.1175/JAS-D-19-0080.1.

Martin, J. E., 2006: Mid-Latitude Atmospheric Dynamics: A First Course. John Wiley and Sons, 324 pp.

Moncrieff, M. W., and M. J. Miller, 1976: The dynamics and simulation of tropical cumulonimbus and squall lines. Quart. J. Roy. Meteor. Soc., 102, 373-394, https://doi.org/10.1002/qj.49710243208.

Pauluis, O., 2008: Thermodynamic consistency of the anelastic approximation for a moist atmosphere. J. Atmos. Sci., 65, 2719-2729, https://doi.org/10.1175/2007JAS2475.1.

Schubert, W. H., S. A. Hausman, M. Garcia, K. V. Ooyama, and H.-C. Kuo, 2001: Potential vorticity in a moist atmosphere. J. Atmos. Sci., 58, 3148-3157, https://doi.org/10.1175/15200469(2001)058<3148:PVIAMA > 2.0.CO;2.

Skamarock, W. C., and Coauthors, 2008: A description of the Advanced Research WRF version 3. NCAR Tech. Note NCAR/TN-475+STR, 113 pp., https://doi.org/10.5065/D68S4MVH

Smith, L. M., and S. N. Stechmann, 2017: Precipitating quasigeostrophic equations and potential vorticity inversion with phase changes. J. Atmos. Sci., 74, 3285-3303, https://doi.org/10.1175/ JAS-D-17-0023.1.

Stechmann, S. N., and A. J. Majda, 2006: The structure of precipitation fronts for finite relaxation time. Theor. Comput. Fluid Dyn., 20, 377-404, https://doi.org/10.1007/s00162-006-0014-1.

Stevens, B., 2005: Atmospheric moist convection. Annu. Rev. Earth Planet. Sci., 33, 605-643, https://doi.org/10.1146/ annurev.earth.33.092203.122658.

Wetzel, A. N., L. M. Smith, S. N. Stechmann, and J. E. Martin, 2019: Balanced and unbalanced components of moist atmospheric flows with phase changes. Chin. Ann. Math., 40B, 1005-1038, https://doi.org/10.1007/s11401-019-0170-4. 